\title{
Abstract (185)
}

High grade serous ovarian cancer (HGSOC) is the most frequent type of ovarian cancer. Most patients have primary response to platinum-based chemotherapy but frequently relapse, which leads to patient death. A lack of well documented and characterized patient-derived HGSOC cell lines is so far a major barrier to define tumor specific therapeutic targets and to study the molecular mechanisms underlying disease progression. We established 34 patient-derived HGSOC cell lines and characterized them at cellular and molecular level. Particularly, we demonstrated that a cancer-testis antigen PRAME and Estrogen Receptor could serve as therapeutic targets. Notably, data from the cell lines did not demonstrate acquired resistance due to tumor recurrence that matched with clinical observations. Finally, we presented that all HGSOC had no or very low CDKN1A (p21) expression due to loss of wild-type TP53, suggesting that loss of cell cycle control is the determinant for tumorigenesis and progression. In conclusion, patient-derived cell lines reveal that PRAME is a potential tumor specific therapeutic target in HGSOC and counteracting the down-regulation of p21 caused by loss of wild-type TP53 might be the key to impede disease progression. 


\title{
Title: Patient-derived cell line models revealed therapeutic targets and molecular mechanisms underlying disease progression of high grade serous ovarian cancer
}

\begin{abstract}
Authors: Caroline Kreuzinger ${ }^{\mathrm{a}+}, 1$, Isabel von der Decken ${ }^{\mathrm{a}+}, 1$, Andrea Wolf ${ }^{\mathrm{a}}$, Magdalena Gamperl $^{\mathrm{a}}$, Julia Koller ${ }^{\mathrm{a}}$, Jasmine Karacs ${ }^{\mathrm{a}}$, Stephanie Pfaffinger ${ }^{\mathrm{a}}$, Thomas Bartl ${ }^{\mathrm{a}}$, Alexander Reinthaller $^{\mathrm{a}}$, Christoph Grimm ${ }^{\mathrm{a}}$, Christian F Singer $^{\mathrm{b}}$, Elena Ioana Braicu ${ }^{\mathrm{c}, \mathrm{d}}$, Paula Cunnea ${ }^{\mathrm{e}}$, Charlie Gourley ${ }^{\mathrm{f}}$, Dominiek Smeets ${ }^{\mathrm{g}, \mathrm{h}}$, Bram Boeckx $^{\mathrm{g}, \mathrm{h}}$, Diether Lambrechts ${ }^{\mathrm{g}, \mathrm{h}}$, Paul Perco, Reinhard Horvat ${ }^{\mathrm{j}}$, Els M. J. J. Berns ${ }^{\mathrm{k}}$, Dan Cacsire Castillo-Tong ${ }^{\mathrm{a}^{\mathrm{k}}}$

$+: \underline{1:}$ These authors contribute equally to the manuscript.
\end{abstract}

\section{Affiliations:}

${ }^{\mathrm{a}}$ Translational Gynecology Group, Department of Obstetrics and Gynecology, Comprehensive Cancer Center, Medical University of Vienna, 1090 Vienna, Austria;

${ }^{\mathrm{b}}$ Department of Gynecology and Gynecologic Oncology, Gynecologic Cancer Unit, Comprehensive Cancer Center, Medical University of Vienna, 1090 Vienna, Austria;

${ }^{\mathrm{c}}$ Tumor Bank Ovarian Cancer Network, Department of Gynecology, Charité Universitätsmedizin Berlin, 13353 Berlin, Germany;

${ }^{\mathrm{d}}$ Department of Gynecology, Charité Universitätsmedizin Berlin, 13353 Berlin, Germany;

${ }^{\mathrm{e}}$ Ovarian Cancer Action Research Centre, Department of Surgery and Cancer, Imperial College

London, London W12 0HS, United Kingdom; 
${ }^{\mathrm{f}}$ Nicola Murray Centre for Ovarian Cancer Research, Cancer Research UK Edinburgh Centre, MRC IGMM, University of Edinburgh, Western General Hospital, Edinburgh EH4 2XR, United Kingdom;

${ }^{\mathrm{g}} \mathrm{KU}$ Leuven, Department of Human Genetics, Laboratory for Translational Genetics, 3000 Leuven, Belgium;

${ }^{\mathrm{h}}$ VIB, VIB Center for Cancer Biology, Laboratory for Translational Genetics, 3000 Leuven, Belgium;

${ }^{\mathrm{i}}$ Emergentec Biodevelopment GmbH, 1180 Vienna, Austria; Current affiliation: Medical University Innsbruck, Department of Internal Medicine IV, 6020 Innsbruck, Austria;

${ }^{\mathrm{j}}$ Department of Clinical Pathology, Medical University of Vienna, 1090 Vienna, Austria;

${ }^{\mathrm{k}}$ Department of Medical Oncology, Erasmus MC Cancer Institute, 3000 CA Rotterdam, The Netherlands

*Correspondence: Dan Cacsire Castillo-Tong, Ebene 5Q, AKH; Waehringer Guertel 18-20, Translational Gynecology Group, Department of Obstetrics and Gynecology, Medical University of Vienna, 1090 Vienna, Austria

Email: dan.cacsire-castillo@meduniwien.ac.at 


\section{Abstract (180184 words)}

High grade serous ovarian cancer (HGSOC) is the most frequent type of ovarian cancer. Most patients have primary response to standard-platinum-based chemotherapy but frequently relapse with resistant disease, which leads to patient death. The 5 year survival rate is below $40 \%$. In addition, due to unspecific killing of cells by the drug, the patients suffer from severe side effects. A lack of well documented and characterized patient-derived HGSOC cell lines has beenis so far a major barrier to define tumor specific therapeutic targets and to study the molecular mechanisms underlying disease progression. We established 34 patient-derived HGSOC cell lines and characterized them at the cellular and molecular level. Particularly, we demonstrated that a cancer-testis antigen PRAME and the Estrogen Receptor could serve as therapeutic targets.

Notably, data from the cell lines did not demonstrate acquired resistance due to tumor recurrence that matched with clinical observations. Finally, we presented that all HGSOC had no or very low CDKN1A (p21) expression due to loss of wild--type TP53, suggesting that loss of cell cycle control is the determinant for tumorigenesis and progression. In conclusion, patient-derived cell lines reveal that PRAME is a potential tumor specific therapeutic target in HGSOC and counteracting the down-regulation of p21 caused by loss of wild-type TP53 might be the key to impede disease progression.

Key words: high grade serous ovarian cancer; patient derived cell lines; acquired platinum resistance; PRAME; therapeutic targetstumor specific antigen; TP53; p21 down-regulation 


\section{Introduction}

Epithelial ovarian cancer (EOC) is a rare disease with a worldwide incidence and mortality of 240000 and 150000 , respectively [1]. $75 \%$ of EOC are high grade serous ovarian cancers (HGSOC) that are often diagnosed at advanced stage. Standard of care includes debulking surgery and platinum-based therapy. Most patients show primary response; however the vast majority relapses with resistant disease, which is defined as acquired resistance. Tumor cell proliferation cannot be controlled and patients die because of disease progression. The 5-year survival rate of patients with advanced HGSOC is below 40\%. Resistance is considered the major course of the patients' death [2]. In addition, platinum-based drugs kill large numbers of nontumor cells, dramatically reducing patients' quality of life [3]. In order to improve ovarian cancer treatment, tumor specific targets have to be defined for the development of more efficient and less toxic therapies.

At the molecular level, common features of HGSOC include TP53 mutations (96\%) and loss of homologous recombination repair (HRR) due to mutational or epigenetic inactivation of the $B R C A 1 / 2$ or other HRR genes [4]. Aside from these common traits, HGSOCs are highly heterogeneous with large amounts of copy number changes and chromosomal rearrangements. The genetic instability and the loss of functional p53 and BRCA1/2 have a direct consequence of higher number of mutations in tumor cells.

To better understand the mechanisms underlying the resistance of HGSOC, well characterized experimental models representing the cellular and genetic backgrounds of HGSOC are essential. Unfortunately, by comparing copy number changes, mutations and mRNA profiles with tumors, it was discovered that the cell lines historically used for ovarian cancer research were most unlikely to be representative of HGSOC [5, 6]. For this reason, several groups including ourselves have previously attempted to establish HGSOC cell lines [6-11]. The number of the 
cell lines generated from these studies is rather low and with no systematic molecular analyses. The lack of linked clinical data about the donors is also a drawback to understand the impact of cellular and molecular characteristics on disease progression [12].

To address this challenge, we established 34 cell lines from 23 HGSOC patients and analyzed the acquired resistance in these tumors and the molecular mechanisms of disease progression. Furthermore, we investigated potential therapeutic targets using these models and validated the results using RNA sequencing data from 66 paired primary and recurrent HGSOC tumors published in a previous study [13]. The cell line models reveal that a cancer-testis antigen PRAME is a potential therapeutic target for HGSOC and the down regulation of p21 caused by the loss of wild-type TP53 is the most important event for disease progression. 


\section{Materials and methods}

2.1. Establishing and maintaining HGSOC cell lines and primary mesothelial cell culture The methods of establishing and maintaining cell lines were published previously [11]. For tumor tissues, we abandoned the previous enzymatic digestion because of a very low success rate and used a cell scraper to release the tumor cells, which were collected by centrifugation. In some ascites, many mesothelial cells grew quickly covering the surface between tumor cell clusters in the culture flasks at the beginning passages. They detached very quickly upon trypsinization ( $<2$ mins), while tumor cells needed longer to detach ( $>5 \mathrm{mins})$. Using selective trypsinization, we were able to isolate primary cell culture that predominantly contains mesothelial cells from tumor cells.

To authenticate the cell lines to patients, DNA finger printing was determined with PowerPlex 21 PCR Kit (Fitchburg, WI, USA), including 21 loci D1S1656, D2S1338, D3S1358, D5S818, D6S1043, D7S820, D8S1179, D12S391, D13S317, D16S539, D18S51, D19S433, D21S11, Amelogenin, CSF1PO, FGA, Penta D, Penta E, TH01, TPOX, and vWA [14] and compared to that of the germline DNA.

\subsection{Extraction of DNA and RNA and cDNA synthesis}

DNA and RNA were extracted with AllPrep DNA/RNA Mini Kit (QIAGEN, Venlo, The Netherlands). DNA from formalin-fixed, paraffin-embedded (FFPE) tissue and blood samples was isolated using QIAamp DNA FFPE Tissue Kit (QIAGEN) and QIAamp DNA Mini Kit (QIAGEN). The quantity was determined by measuring the absorbance at 260/280 nm with Nanodrop 1000 (Thermo Fisher Scientific, Massachusetts, USA) and the quality of RNA was controlled using Agilent RNA 6000 Nano Kit (Agilent Technologies, CA, USA) and Agilent 
2100 Bioanalyzer (Agilent Technologies). Samples with an RNA Integrity Number $>8$ were further processed for RNA sequencing. cDNA was synthesized with Omniscript RT Kit (Qiagen).

2.3. Detection of mutations in the coding region of TP53, KRAS and BRCA1/2 Alterations in the coding regions of TP53 and KRAS genes were examined by Sanger sequencing. For TP53, three DNA fragments C0 (sense: 5'-TGCTTTCCACGACGGTGAC-3'; antisense: 5'AGCAGCCTCTGGCATTCTG-3'), C1 (sense; 5'-CCTCCTCAGCATCTTATC-3', antisense: 5'-AAGAAGTGGAGAATGTCAG-3') and C2 (sense: 5'-CCAAGCAATGGATGATT-3'; antisense: 5'-TAGTGGATGGTGGTACAGTC-3') covering exon 1-4, 4-7 and 6-11, were amplified with annealing temperatures of $55^{\circ} \mathrm{C}, 50^{\circ} \mathrm{C}$ and $52^{\circ} \mathrm{C}$, respectively. For $K R A S$, one single fragment covering the whole coding region (sense: 5'-ATTTCGGACTGGGAGCGAG-3'; antisense: 5'-GCATCATCAACACCCAGATTAC-3') was amplified with an annealing temperature of $60^{\circ} \mathrm{C}$ with cDNA samples. PCR was performed with MangoMix (Bioline Reagent Ltd, London, UK) with 40 cycles of $30 \mathrm{sec}$ at $94^{\circ} \mathrm{C}, 30 \mathrm{sec}$ at the corresponding annealing temperature, and $30 \mathrm{sec}$ at $72^{\circ} \mathrm{C}$. The reactions were preceded with a 10 min denaturation step and an extension at $72^{\circ} \mathrm{C}$ for $10 \mathrm{~min}$. Mutations in the $B R C A 1$ and $B R C A 2$ coding regions were detected using TruSeq Custom Amplicon Design - AFP2, TruSeq CustomAmplicon Index Kit and Reagent Kit v2 (300cycles) and Illumina MiSeq System (Illumina; Inc., CA, USA). Once an alteration was found, it was verified with DNA or cDNA samples and confirmed in cell lines at different passages by Sanger sequencing.

2.4. Measurement of mutant DNA proportion by allele specific digital droplet PCR (ddPCR) Allele specific ddPCR systems were designed to detect the absolute copy numbers of wild type and mutant TP53 and KRAS in DNA samples. Primers and probes are shown in Supplementary Table S4. PCR was performed using ddPCR Supermix for Probes (Bio-Rad Laboratories, Inc., 
CA, USA) according the manufacturer's instruction. Signals were detected by QX200 Droplet Digital PCR System (Bio-Rad Laboratories, Inc.). We used these systems to determine the tumor cell proportion in cell culture. Additionally, we performed these assays for cell lines at higher passages to ensure that all cells harbored their specific mutations.

2.5 Low coverage whole genome sequencing and RNA-sequencing and annotation

The methods were described in detail previously [11].

\subsection{Determination of DNA copy number}

DNA copy number of TP53 and BRCA1/2 were detected with the corresponding PrimPCR ddPCR Copy Number Assays (dHsaCP1000586, dHsaCP2500367, dHsaCP2500368, respectively, Bio-Rad, CA, USA) with EIF2C1 (dHsaCP2500349) as reference gene following the manufacturer's instruction (Bio-Rad Laboratories, Inc.). DNA from a healthy individual was used as a control.

\subsection{Unsupervised clustering}

RNA sequencing data includes 63 samples from 33 cell lines with one line 17066 excluded because of bad quality of sequencing results. Euclidean distance clustering was performed on the EDA normalized data using the top $10 \%$ of the expressed genes ranked by their standard deviation across the cohort.

\subsection{Analyses of specific gene expression}

Since cell lines with low passages had higher possibility to contain other types of cells, some of the samples were excluded according to the results of unsupervised clustering (indicated in 
Figure 1). Mean expression was used for cell lines with more than one measurements at higher passages.

\subsection{In vitro carboplatin sensitivity test}

Chemo-sensitivity was measured by MTT assay using EZ4U-kit following the manufacturer's instructions (Biomedica, New Hampshire, USA). $7 \times 10^{3}$ cells per well were seeded in 96-well plates (Costar, Corning Incorporated, USA) and incubated with carboplatin (Accord Healthcare, North Harrow, UK) in triplicate at $37^{\circ} \mathrm{C}$ and $5 \% \mathrm{CO}_{2}$ for $96 \mathrm{~h}$. At least three independent experiments were performed for each cell line. $\mathrm{IC}_{50}$ values were calculated using a non-linear dose-response curve with sigmoidal fit of logarithmic mean across the triplets of each assay by GraphPad Prism 5.00 for Windows (GraphPad Software, San Diego, CA, USA; www.graphpad.com). Carboplatin concentrations ranging from $100 \mu \mathrm{g} / \mathrm{ml}(270 \mathrm{M})$ to $0.4 \mu \mathrm{g} / \mathrm{ml}$ $(1.08 \mu \mathrm{M})$ with 1:2 serial dilution between them were used in the initial test. We chose this range of concentrations, as the plasma peak value of carboplatin used for patients is around $300 \mu \mathrm{M}$. If the cells showed borderline values of sensitivity, higher or lower concentrations were included in additional tests.

\subsection{Comparison of gene expression in two different groups of cell lines}

We compared the gene expression in tumor cells established before and after exposing to carboplatin-based therapy and tumors with or without wild-type $B R C A 1 / 2$. For the latter, means were calculated in case more than one cell line was established from the same patient. The nbinomTest function of the DEseq R package was used after normalization for the identification of differentially expressed transcripts [15]. p-values were adjusted using Benjamini Hochberg methods thus controlling the false discovery rate (FDR). Transcripts with an FDR $<5 \%$, a fold- 
change value $>|5|$, and read counts $>50$ in the group showing higher read counts were considered as relevant.

\subsection{Selection of potential tumor specific antigens}

We selected genes coding for potential tumor specific antigens mainly from four sub-groups: (i) Cancer-testis like antigens (CTA); (ii) Differentiation antigens; (iii) Oncofetal antigens including $\alpha$-fetoprotein, carcinoembryonic antigen (CEA), trophoblast glycoprotein, onco-trophoblast, and solid tumor associated glycoprotein; (iv) Over-expressed antigens, mainly based on the information provided by Even-Desrumeaux et al. [16, 17]. Particularly, for CTAs, we referred the 118 genes described as "testis-selective" or "testis restricted" in the CTDatabase maintained by The Ludwig Institute for Cancer Research (http://www.cta.lncc.br/).

\subsection{Measurement of PRAME gene expression by RT-qPCR}

TaqMan Assay Gene Expression Hs01022301_m1 was used to measure the PRAME expression in cell lines according to manufacturer's instructions. A cell line with relative high expression of PRAME (according to RNA sequencing data) was used to make a dilution series to generate a standard curve. Relative expression values were calculated in fold of the standard samples. Cell line data were median-normalized for comparison of RT-qPCR results with RNA sequencing results.

\subsection{Immunohistochemistry staining and immunofluorescent staining}

Immunohistochemistry was performed as described previously [11]. Antibodies against PRAME (PA5-13679; Thermo Fischer Scientific) and EpCAM (ab32329; abcam, Cambridge, UK) was diluted at 1:400 and 1:500 in Dako Antibody-Diluent (Agilent Technologies), respectively, and incubated with the slides at $4^{\circ} \mathrm{C}$ overnight. Immunofluorescent staining (IF) was performed on 
slides pretreated with adhesion substance (Paul Marienfeld GmbH \& Co. KG, LaudaKönigshofen, Germany). 50000 cells were subjected to each well on the slides following the instruction of the manufacturer, dried and fixed in $4 \%$ paraformaldehyde. The slides were treated with $0.5 \%$ Triton X-100 in PBS, washed 3 times with PBS, each time for 3 mins and incubated with Dako Ultra Vision Block (Thermo Fischer Scientific) for 7 min at RT followed by washing in PBS-Tween 20 twice, each for $3 \mathrm{~min}$. The PRAME antibody was diluted at 1:100 in Dako Antibody-Diluent and incubated at $4^{\circ} \mathrm{C}$ overnight. Alexa Fluor 488 goat anti-Rabbit IgG (Thermo Fisher Scientific) was diluted 1:500 in Dako Antibody-Diluent and incubated for an hour at RT. DAPI staining was performed with standard protocol and the slides were mounted with Fluoromount-G (Southern Biotech, Birmingham, USA).

\subsection{Data and materials availability}

We are willing to distribute any materials (cell lines), data (RNA sequencing, low coverage sequencing, patient clinicopathological data) and protocols in the published experiments to qualified researchers for their own use upon reasonable request. 


\section{Results}

\subsection{HGSOC cell lines and patients}

34 tumor cell lines from ascites, tumor tissue and pleural fluid from 23 patients with HGSOC were established (Table 1). Part of the results regarding seven of them was reported previously [11].

From five patients (P3, P9, P12, P16, P17), we established more than one cell line from tumor material collected either from different locations or at different time points (Table 1). We also cultivated primary mesothelial cells from the ascites of eight patients, which could be passaged approximately 10 times until they underwent senescence or died. Their cell type identity was confirmed by pathologists (Supplementary Figure S1). In addition, they expressed very high level of CALB2 (>4000 read counts), which is a marker for mesothelial cells, and had little or no expression of EPCAM (26-692 read counts). This was in contrast to tumor cells, which had very high EPCAM expression (1781-47851 read counts) and no or very low CALB2 expression (0-304 read counts). Furthermore, they had wild-type TP53, while their counterpart tumor cells harbored mutant TP53 (see also 3.2).

All cell lines were authenticated to be from the corresponding patients by DNA finger printing analyses (Supplementary Table S1). The purity of the tumor cells was determined by allele specific digital droplet PCR systems measuring the proportions of tumor specific mutant TP53 or KRAS DNA copies. All cell lines have been passaged over 30 times.

\subsection{The cell lines are stable experimental models for HGSOC}

In all cell lines except one, unique TP53 alterations affecting the transcripts were detected and no wild type allele could be found (Table 1, Supplementary Table S2). The alterations in mRNA were derived either directly from DNA or from intronic changes leading to variant splicing. p53 
protein was truncated with variant C-terminals or affected by substitution or deletion of amino acids. All TP53 alterations were located in the DNA binding domain except in patients P4 and P6, whose tumors had alterations in the oligomerization domain (Supplementary Figure S2). Germline BRCA1 and BRCA2 mutations were detected in one (P6) and three (P5, P7, P11) of these cell lines, respectively, all losing wild-type alleles in tumor cells. Patient P17 presented with a somatic BRCA2 mutation with loss of heterozygosity. In addition, P15 had a heterozygous germline $B R C A 2$ mutation, which was retained in the corresponding tumor. The tumor cells from P18 harbored a heterozygous somatic BRCA2 mutation. The only TP53 wild-type cell line 8540 had a heterozygous KRAS mutation. We re-examined two FFPE blocks and confirmed the high grade serous histological type (Supplementary Figure S3). The genetic profile of the FFPE samples, the cell lines and the patient blood sample were confirmed by DNA finger printing analyses. Both mutant and wild-type DNA copies of $K R A S$ were detected in DNA samples isolated from FFPE sections by allele specific ddPCR. Sanger Sequencing confirmed that the status of TP53, BRCA1/2 and KRAS mutations was unchanged in different cell lines established from the same patients and remained stable through passaging.

Low coverage sequencing revealed that all TP53 mutant cell lines had a high incidence of copy number alterations (CNA) compared to their corresponding germline DNA, e.g. P_16099B. The TP53 wild-type and KRAS mutant cell line P8_8540 had less CNA than the TP53 mutant cell lines (Supplementary Figure S4). Nevertheless, cell lines established from the same patients presented similar pattern of CNA.

Unsupervised clustering of gene expression profiles of 63 RNA samples including cell lines with different passages (Figure 1) revealed that (i) the primary mesothelial cell cultures had different expression profiles than all tumor cell lines; (ii) different passages of the same tumor cell lines had very similar expression profiles; (iii) cell lines derived from the same patient had the most similar expression profiles, regardless of whether they were derived from different locations (e.g. 
Patient P17) or whether they were established at different time points of disease progression (e.g. Patients P3, P9, and P12).

3.3. Cell cycle pathway is the determinant for tumorigenesis and tumor progression We first examined genes, whose transcription is directly regulated by p53 and found that major genes controlling cell cycle arrest, apoptosis, survival and senescence $C D K N 1 A(p 21), B A X$, TIGAR, PAI1 and MDM2 were all down-regulated in the TP53 mutant tumor cells in comparison with the TP53 wild type cell line 8540 and the mesothelial cells (Figure 2A-E). Furthermore, cyclins controlling the cell cycle restraint points cyclin D1 (CCND1), E1 (CCNE1), A1 (CCNA1) and $B 2(C C N B 2)$ were overexpressed in all tumor cells. No obvious differences could be observed regarding the expression of other cyclins (Figure 2F-O). The KRAS mutant cell line 8540 showed additional higher expression of cyclin D2 (CCND2) (Figure 2G) in comparison to all other cell lines.

\subsection{Response to carboplatin in vitro and in vivo}

Most of the tumor cell lines showed lower $\mathrm{IC}_{50}$ values for carboplatin than the primary mesothelial cell cultures, indicating that carboplatin preferentially kills tumor cells (Figure 3A). A few cell lines showed very high values $(8684,18483)$. Different cell lines from the same patients presented different pattern of changes of the $\mathrm{IC}_{50}$ values. Some became more resistant in later established lines (e.g. 15233_nov was collected 12 months later than 13363; 17249 was collected 20 days later than 17142), while others showed the opposite change and became more sensitive (e.g. the 6 cell lines from P9 were derived from ascites isolated over a total interval of 12 months; EK_R1 was taken 6 months later then 8714 and 17457). Cell lines from P17 were established from ascites $(17480)$ and tumor tissues $(8715,8716)$ at primary diagnosis and showed similar sensitivities. 
Seven patients had no residual tumors after primary surgery, presented a clinical complete remission at the completion of the first line treatment, and then developed recurrent disease. From six of these patients, we established cell lines from primary tumor material. By comparing the $\mathrm{IC}_{50}$ value with the progression free interval (PFI), no correlation could be observed between the two parameters (Figure 3B).

We further examined clinical data of all patients (Supplementary Figure S5). 10 patients had at least one recurrence (red colored) and received at least 2 lines of platinum-based treatment. The CA-125 dropped upon the treatment of platinum-based drug by almost all cycles with a few exceptions during the whole treatment.

In addition, we did not observe particularly low $\mathrm{IC}_{50}$ values of the cell lines with $B R C A 1$ or BRCA2 mutations (Figure 3A). By comparing gene expression profiles of the cell lines from the 5 patients with only mutant $B R C A 1 / 2$ and the remaining 18 patients with at least one wild type $B R C A 1 / 2$ allele, we did not find any differentially expressed genes between the two groups, even after lowering the fold change (FC) threshold to 2.

Moreover, we compared gene expression profiles of cell lines with and without exposure to chemotherapy treatment and we found that only one gene $N B L 1$ was overexpressed in the exposed cells and eight transcripts were overexpressed in the naive cells, all being non-coding RNA genes (RP5-1114G22.2, FAM103A2P, OVAAL, RP11-527N22.1, DDX11L9, RP11438N16.1, H19, AC004540.4). By lowering the fold change (FC) threshold from 5 to 2, the results were unchanged.

3.6. PRAME and the estrogen receptor (ER) are potential therapeutic targets for HGSOC We selected a panel of tumor associated antigens including cancer-testis like antigens, differentiation antigens, oncofetal antigens and some known cancer related overexpressed antigens $[16,17]$ and examined their expression in tumor cell lines in comparison with the five 
mesothelial cell cultures. Most of the genes had no or little expression in all samples (Supplementary Table S3; AFP; BAGE2; CEACAM3, 4, 5, 7, 8, 16, 18, 20, 21; CEACAMP1, 2, 5, 7, 8, 9, 11; MAGEA3, A12, B2, C1; MUC2, 3A, 5AC, 12, 13, 19; PSG1; TEX15; TLX1, 2, 3). Some had comparable high expression in both tumor cells as well as in the mesothelial cells (e.g. EGFR, ERBB2, MGAT5, MUC16, SPAG9, TPBG, TSPYL1). 27 genes were found to be higher expressed in tumor cells than in mesothelial cells, but with different prevalence. Three had low expression in tumors and high expression in mesothelial cells and 16 had rather low prevalence of highly expressing tumors (Table 2). Four genes MAGEA4, MAGEA11, MUC4 and SPAG1 had high expression in less than $50 \%$ of the samples. Four genes had high prevalence in all tumors, among which FOLRI, MUC1 and MUC20 are expressed in multiple organs/tissues, such as kidney, salivary gland, lung, or fallopian tube (https://www.proteinatlas.org; https://www.genecards.org). PRAME was highly expressed in $94 \%$ of all tumor cell lines but not in the mesothelial cells. It was reported to be only expressed in testis tissue and to a lesser extent in ovary $[18,19]$. PRAME was not expressed in 8540 and had a very low expression in 18605 (Figure 4A). Evaluation of the RNA sequencing data with RT-qPCR confirmed that PRAME indeed had very high expression in 31/33 cell lines and was not expressed in mesothelial cells and fibroblasts (Figure 4B). By examining the RNA sequencing data from 66 matched primary and recurrent HGSOC tumor tissues from a previous study [13], high expression of PRAME was confirmed (Figure 4C) in almost all tumors. A few samples showed lower expression in both samples or in one of the matched samples (Figure 4D).

Immunohistochemistry and immunofluorescent staining of the corresponding tumor tissues showed that PRAME protein was expressed in nucleus, cytoplasm and at the membrane of the tumor cells (Figure 5).

Additionally, the gene coding for ER, ESR1 showed higher expression in TP53 mutant tumor cells and was low in 8540 and the mesothelial cells (Figure 2P). There was no or very low 
expression of the progesterone receptor $(P G R)$ in TP53 mutant tumor cells (read counts:

median=1, q1=0, q3=23) and 8540 (read count: 3$)$. 


\section{Discussion}

We successfully established 34 patient-derived HGSOC cell lines and characterized them at the cellular and molecular level. With these new cell lines, we provide useful models to the scientific community to study the tumorigenesis and progression of HGSOC. Particularly, we demonstrated that a cancer-testis antigen PRAME and the ER could serve as therapeutic targets. The experimental results did not show acquired resistance of recurrent tumors, which was in line with the clinical observations. Finally, we presented that all HGSOC had no or very low $C D K N 1 A$ (p21) expression due to loss of wild type TP53.

Since Domcke et al. [5] pointed out that many of the ovarian cancer cell lines historically used for research are unlikely to be of high grade serous origin, great efforts have been paid to establish patient-derived new models including cell lines and patient-derived xenografts. The current study presents 34 new cell line models, analyzed at the level of transcriptomics, CNV profile and mutations of TP53, BRCA1/2 and KRAS genes that are linked to clinicopathological information, treatment, and response of the patients.

Despite extensive research and the introduction of some additional therapeutic options such as Bevacizumab, ovarian cancer remains a cancer with poor prognosis [20]. Carboplatin is still the gold standard treatment for HGSOC, which kills cells nonspecifically and is highly toxic [3]. So far, identifying tumor specific targets has been a big challenge for the scientific community. Some proteins, such as the Wilms tumor protein, could not serve as an optimal target, since they are not only highly expressed in ovarian cancer cells, but also in the mesothelium [21]. An ideal therapeutic target should have a homogeneous expression in tumors and no or minimal expression in normal tissues [16]. Thus, we examined the expression levels of a panel of genes coding for possible tumor associated antigens and some membrane proteins. Four genes, MUC1, MUC20, FOLRI and PRAME with high expression levels and high prevalence across all tumor 
cell lines were identified. Among these four genes, $M U C 1$ is expressed in almost all epithelial tissues and in some hematopoietic cells [22] and MUC20 was detected in multiple organs, such as kidney, lung, liver, and the digestion system [23]. Expression of FOLRI was also found in normal tissues such as kidney, bladder, salivary glands, lung and some cells of the nerve system [24]. In contrast to the multiple tissue expression of these three genes, PRAME expression is only found in normal testis tissues and to a lesser extent in the ovary. Since the ovaries of the HGSOC patients were affected by tumors and are usually removed during surgery, targeting PRAME would not affect normal tissues. Our results also showed that $P R A M E$ was not expressed in mesothelial cells lining the peritoneal cavity and in fibroblasts, which makes PRAME an optimal target for therapy development.

PRAME, the preferentially expressed antigen in melanoma gene, was first detected in a mRNA study of human testis [25]. It codes a membrane-bound protein and triggers autologous cytotoxic T cell-mediated immune responses, presumably through retinoic acid signaling [18, 26, 27]. Its overexpression in several solid tumors, such as melanoma [28], breast cancer [19], and sarcomas $[29,30]$ as well as in hematological malignancies [27] makes it an interesting therapeutic target. Recent studies using recombinant PRAME and an immunostimulant demonstrated a cellular immune response in patients in addition to safety [31, 32]. Furthermore, a polyclonal antibody was produced against PRAME and was shown to bind recombinant PRAME ex vivo as well as different cells in vitro [33]. In ovarian cancer, PRAME was found to be frequently expressed in epithelial cancer at both mRNA and protein levels, which was regulated by DNA methylation [34], and to have prognostic value for stage III serous cancer [35-37] . Further investigation will be needed to understand the expression activation and functions of PRAME in TP53 mutant HGSOC to provide the basis for drug development, for which the new patient-derived cell lines presented in this study are ideal models. 
Patients with serous ovarian cancer that have positive hormonal receptor status were shown to have worse survival than those with negative receptor status [38]. Since the ovary produces estrogen, which could promote cell growth upon binding to its receptor ER, we also examined the expression level of ESRl gene and found a high expression of this gene in most of the TP53 mutant tumor cell lines. Endocrine therapy was shown to bring benefits to patients with advanced epithelial ovarian cancer [39], probably by blocking the ESR1 promoted tumor cell growth. Even though the effect was not specified to HGSOC, it is worth further investigation in a well-defined population. Using our cell line models, the transcriptional regulation of ESR1 could be further studied.

Originally, platinum resistance was defined for tumors, which recur within 6 months after completing first line therapy [40]. This concept of clinical resistance, however, does not necessarily associate with the response of tumor cells to drug. Other possible reasons for a rapid recurrence of tumors could be the quick exponential proliferation or/and higher number of living tumor cells after therapy due to poor accessibility of the drug. This clinical definition of resistance was equaled to a cellular resistance in 2011 for ovarian cancer [41], while the hypotheses of acquired resistance and the evolution of resistance were proposed. Our cell line models showed that recurrent tumor cells did not become more "resistant" to carboplatin than their primary counterparts, which was in accordance with patient response presented by clinical data, suggesting that recurrent tumors resemble their primary ancestors. Molecular analyses of their transcriptional profiling and $\mathrm{CNV}$ profiles further suggest the similarities of the primary and recurrent tumor cells. Nevertheless, the current study included a small cohort of 23 patients and establishing cell lines might involve selections of tumors with distinct molecular characteristics. Thus, evaluation in a larger cohort of patients will be needed to clarify this issue. By examining genes, whose transcription is directly regulated by p53 [42], a clear down regulation was observed for the key genes, which regulate the cell cycle (p21, CNKN1A), 
apoptosis $(B A X)$, senescence (PAI1) [43] and survival (TIGAR) [44] in all TP53 mutant cell lines, indicating that the loss of wild-type p53 functions is the major driving force of tumor cell progression. We also observed higher expression of genes coding for cyclins D1 (CCND1), E1 (CCNE1), A1 (CCNA1) and B2 (CCNB2), demonstrating that TP53 mutant tumor cells had a high endogenous level of all cyclins needed to pass the G1/S and G2/M restriction points. Synchronized with the down-regulation of $\mathrm{p} 21$, the inhibitor of the cyclin and the cyclindependent kinase complexes, tumor cells lost the restraints in their cell cycle and were directed to proliferation. Our data suggest that loss of the wild-type TP53 is not only the reason for tumorigenesis but also the driver of tumor progression. Although well established, we confirmed in the current study that all these cellular processes are common for all TP53 mutant HGSOC. It is important to emphasize on the determinant role of cell cycle control in HGSOC, so that efforts could be directed towards the main reason of disease progression in the development of new therapies.

Taken together, we (i) established and molecularly analyzed 34 cell lines from 23 HGSOC patients, (ii) showed that loss of TP53 wild type was the main driving force of tumorigenesis and tumor progression, and (iii) identified $P R A M E$ as a potential therapeutic target. The cell line models can be applied for therapy development in addition to the investigation of molecular mechanisms in disease progression.

\section{Acknowledgements}

This work was supported by European Community’s Seventh Framework Programme under grant agreement No. 279113-2 (OCTIPS; www.octips.eu). All authors declare that we have no financial conflict of interest that might be construed to influence the results or interpretation of the manuscript. 


\section{Author contributions}

Conceptualization, C.K., I.V.D.D., E.I.B., P.C., C.G., D.L., E.M.B, and D.C.C-T; Methodology, C.K., I.V.D.D., A.W., M.G., JA.K., JU.K., S.P., A.R., C.G., C.F.S., D.S., R.H., B.B., and P.P.; Formal Analysis, B.B. and P.P.; Investigation, C.K., I.V.D.D., A.W., M.G., JA.K., JU.K., S.P. and D.C.C-T; Resources, A.R., C.G., T.B. and R. H.; Data curation, C.K., I.V.D.D., A.W., M.G., JA.K., JU.K., S.P., D.L., D.S., and B.B; Writing - original Draft C.K., I.V.D.D. and D.C.C-T; Writing - Review \& Editing, C.K., I.V.D.D., C.G., E.I.B., P.C., C.G., D.S., E.M.B. and D.C.C-T; Visualization, I.V.D.D. and B.B.; Project Administration and Supervision, D.C.C-T; Funding Acquisition, E.I.B., C.G., D.L., E.M.B. and D.C.C.-T. 


\section{References}

[1] D.D. Bowtell, S. Bohm, A.A. Ahmed, P.J. Aspuria, R.C. Bast, Jr., V. Beral, J.S. Berek, M.J. Birrer, S. Blagden, M.A. Bookman, J.D. Brenton, K.B. Chiappinelli, F.C. Martins, G. Coukos, R. Drapkin, R. Edmondson, C. Fotopoulou, H. Gabra, J. Galon, C. Gourley, V. Heong, D.G. Huntsman, M. Iwanicki, B.Y. Karlan, A. Kaye, E. Lengyel, D.A. Levine, K.H. Lu, I.A. McNeish, U. Menon, S.A. Narod, B.H. Nelson, K.P. Nephew, P. Pharoah, D.J. Powell, Jr., P. Ramos, I.L. Romero, C.L. Scott, A.K. Sood, E.A. Stronach, F.R. Balkwill, Rethinking ovarian cancer II: reducing mortality from high-grade serous ovarian cancer, Nat Rev Cancer, 15 (2015) 668-679.

[2] E.M. Berns, D.D. Bowtell, The changing view of high-grade serous ovarian cancer, Cancer Res, 72 (2012) 2701-2704.

[3] M.G. Apps, E.H. Choi, N.J. Wheate, The state-of-play and future of platinum drugs, Endocr Relat Cancer, 22 (2015) R219-233.

[4] N. Cancer Genome Atlas Research, Integrated genomic analyses of ovarian carcinoma, Nature, 474 (2011) 609-615.

[5] S. Domcke, R. Sinha, D.A. Levine, C. Sander, N. Schultz, Evaluating cell lines as tumour models by comparison of genomic profiles, Nat Commun, 4 (2013) 2126.

[6] M.S. Anglesio, K.C. Wiegand, N. Melnyk, C. Chow, C. Salamanca, L.M. Prentice, J. Senz, W. Yang, M.A. Spillman, D.R. Cochrane, K. Shumansky, S.P. Shah, S.E. Kalloger, D.G. Huntsman, Type-specific cell line models for type-specific ovarian cancer research, PLoS One, 8 (2013) e72162.

[7] I.J. Letourneau, M.C. Quinn, L.L. Wang, L. Portelance, K.Y. Caceres, L. Cyr, N. Delvoye, L. Meunier, M. de Ladurantaye, Z. Shen, S.L. Arcand, P.N. Tonin, D.M. Provencher, A.M. Mes-Masson, Derivation and characterization of matched cell lines from primary and recurrent serous ovarian cancer, BMC Cancer, 12 (2012) 379.

[8] Z. Pan, J. Hooley, D.H. Smith, P. Young, P.E. Roberts, J.P. Mather, Establishment of human ovarian serous carcinomas cell lines in serum free media, Methods, 56 (2012) 432-439.

[9] T. Sueblinvong, R. Ghebre, Y. lizuka, S.E. Pambuccian, R. Isaksson Vogel, A.P. Skubitz, M. Bazzaro, Establishment, characterization and downstream application of primary ovarian cancer cells derived from solid tumors, PLoS One, 7 (2012) e50519.

[10] T.A. Ince, A.D. Sousa, M.A. Jones, J.C. Harrell, E.S. Agoston, M. Krohn, L.M. Selfors, W. Liu, K. Chen, M. Yong, P. Buchwald, B. Wang, K.S. Hale, E. Cohick, P. Sergent, A. Witt, Z. Kozhekbaeva, S. Gao, A.T. Agoston, M.A. Merritt, R. Foster, B.R. Rueda, C.P. Crum, J.S. Brugge, G.B. Mills, Characterization of twenty-five ovarian tumour cell lines that phenocopy primary tumours, Nat Commun, 6 (2015) 7419. [11] C. Kreuzinger, M. Gamperl, A. Wolf, G. Heinze, A. Geroldinger, D. Lambrechts, B. Boeckx, D. Smeets, R. Horvat, S. Aust, G. Hamilton, R. Zeillinger, D. Cacsire Castillo-Tong, Molecular characterization of 7 new established cell lines from high grade serous ovarian cancer, Cancer Lett, 362 (2015) 218-228.

[12] F. Jacob, S. Nixdorf, N.F. Hacker, V.A. Heinzelmann-Schwarz, Reliable in vitro studies require appropriate ovarian cancer cell lines, J Ovarian Res, 7 (2014) 60.

[13] C. Kreuzinger, A. Geroldinger, D. Smeets, E.I. Braicu, J. Sehouli, J. Koller, A. Wolf, S. Darb-Esfahani, K. Joehrens, I. Vergote, A. Vanderstichele, B. Boeckx, D. Lambrechts, H. Gabra, G.B.A. Wisman, F. Trillsch, G. Heinze, R. Horvat, S. Polterauer, E. Berns, C. Theillet, D. Cacsire Castillo-Tong, A Complex Network of Tumor Microenvironment in Human High-Grade Serous Ovarian Cancer, Clin Cancer Res, 23 (2017) 76217632.

[14] M.G. Ensenberger, C.R. Hill, R.S. McLaren, C.J. Sprecher, D.R. Storts, Developmental validation of the PowerPlex((R)) 21 System, Forensic Sci Int Genet, 9 (2014) 169-178.

[15] S. Anders, W. Huber, Differential expression analysis for sequence count data, Genome Biol, 11 (2010) R106.

[16] K. Even-Desrumeaux, D. Baty, P. Chames, State of the art in tumor antigen and biomarker discovery, Cancers (Basel), 3 (2011) 2554-2596. 
[17] N. Beauchemin, A. Arabzadeh, Carcinoembryonic antigen-related cell adhesion molecules (CEACAMs) in cancer progression and metastasis, Cancer Metastasis Rev, 32 (2013) 643-671.

[18] H. Ikeda, B. Lethe, F. Lehmann, N. van Baren, J.F. Baurain, C. de Smet, H. Chambost, M. Vitale, A. Moretta, T. Boon, P.G. Coulie, Characterization of an antigen that is recognized on a melanoma showing partial HLA loss by CTL expressing an NK inhibitory receptor, Immunity, 6 (1997) 199-208.

[19] M.T. Epping, A.A. Hart, A.M. Glas, O. Krijgsman, R. Bernards, PRAME expression and clinical outcome of breast cancer, Br J Cancer, 99 (2008) 398-403.

[20] S. Mahner, L. Woelber, V. Mueller, I. Witzel, K. Prieske, D. Grimm, V.A.G. Keller, F. Trillsch, Beyond Bevacizumab: An Outlook to New Anti-Angiogenics for the Treatment of Ovarian Cancer, Front Oncol, 5 (2015) 211.

[21] C. Barcena, E. Oliva, WT1 expression in the female genital tract, Adv Anat Pathol, 18 (2011) 454-465. [22] A.M. Sousa, P.M. Grandgenett, L. David, R. Almeida, M.A. Hollingsworth, F. Santos-Silva, Reflections on MUC1 glycoprotein: the hidden potential of isoforms in carcinogenesis, APMIS, 124 (2016) 913-924. [23] T. Higuchi, T. Orita, S. Nakanishi, K. Katsuya, H. Watanabe, Y. Yamasaki, I. Waga, T. Nanayama, Y. Yamamoto, W. Munger, H.W. Sun, R.J. Falk, J.C. Jennette, D.A. Alcorta, H. Li, T. Yamamoto, Y. Saito, M. Nakamura, Molecular cloning, genomic structure, and expression analysis of MUC20, a novel mucin protein, up-regulated in injured kidney, J Biol Chem, 279 (2004) 1968-1979.

[24] L.E. Kelemen, The role of folate receptor alpha in cancer development, progression and treatment: cause, consequence or innocent bystander?, Int J Cancer, 119 (2006) 243-250.

[25] A. Pawlak, C. Toussaint, I. Levy, F. Bulle, M. Poyard, R. Barouki, G. Guellaen, Characterization of a large population of mRNAs from human testis, Genomics, 26 (1995) 151-158.

[26] N. van Baren, H. Chambost, A. Ferrant, L. Michaux, H. Ikeda, I. Millard, D. Olive, T. Boon, P.G. Coulie, PRAME, a gene encoding an antigen recognized on a human melanoma by cytolytic $T$ cells, is expressed in acute leukaemia cells, Br J Haematol, 102 (1998) 1376-1379.

[27] F. Wadelin, J. Fulton, P.A. McEwan, K.A. Spriggs, J. Emsley, D.M. Heery, Leucine-rich repeat protein PRAME: expression, potential functions and clinical implications for leukaemia, Mol Cancer, 9 (2010) 226. [28] M.G. Field, C.L. Decatur, S. Kurtenbach, G. Gezgin, P.A. van der Velden, M.J. Jager, K.N. Kozak, J.W. Harbour, PRAME as an Independent Biomarker for Metastasis in Uveal Melanoma, Clin Cancer Res, 22 (2016) 1234-1242.

[29] S.M. Pollack, Y. Li, M.J. Blaisdell, E.A. Farrar, J. Chou, B.L. Hoch, E.T. Loggers, E. Rodler, J.F. Eary, E.U. Conrad, 3rd, R.L. Jones, C. Yee, NYESO-1/LAGE-1s and PRAME are targets for antigen specific T cells in chondrosarcoma following treatment with 5-Aza-2-deoxycitabine, PLoS One, 7 (2012) e32165.

[30] P. Tan, C. Zou, B. Yong, J. Han, L. Zhang, Q. Su, J. Yin, J. Wang, G. Huang, T. Peng, J. Shen, Expression and prognostic relevance of PRAME in primary osteosarcoma, Biochem Biophys Res Commun, 419 (2012) 801-808.

[31] J.L. Pujol, T. De Pas, A. Rittmeyer, E. Vallieres, B. Kubisa, E. Levchenko, S. Wiesemann, G.A. Masters, R. Shen, S.A. Tjulandin, H.S. Hofmann, N. Vanhoutte, B. Salaun, M. Debois, S. Jarnjak, P.M. De Sousa Alves, J. Louahed, V.G. Brichard, F.F. Lehmann, Safety and Immunogenicity of the PRAME Cancer Immunotherapeutic in Patients with Resected Non-Small Cell Lung Cancer: A Phase I Dose Escalation Study, J Thorac Oncol, 11 (2016) 2208-2217.

[32] R. Gutzmer, L. Rivoltini, E. Levchenko, A. Testori, J. Utikal, P.A. Ascierto, L. Demidov, J.J. Grob, R. Ridolfi, D. Schadendorf, P. Queirolo, A. Santoro, C. Loquai, B. Dreno, A. Hauschild, E. Schultz, T.P. Lesimple, N. Vanhoutte, B. Salaun, M. Gillet, S. Jarnjak, P.M. De Sousa Alves, J. Louahed, V.G. Brichard, F.F. Lehmann, Safety and immunogenicity of the PRAME cancer immunotherapeutic in metastatic melanoma: results of a phase I dose escalation study, ESMO Open, 1 (2016) e000068.

[33] D. Pankov, L. Sjostrom, T. Kalidindi, S.G. Lee, K. Sjostrom, R. Gardner, M.R. McDevitt, R. O'Reilly, D.L.J. Thorek, S.M. Larson, D. Veach, D. Ulmert, In vivo immuno-targeting of an extracellular epitope of membrane bound preferentially expressed antigen in melanoma (PRAME), Oncotarget, 8 (2017) 6591765931. 
[34] W. Zhang, C.J. Barger, K.H. Eng, D. Klinkebiel, P.A. Link, A. Omilian, W. Bshara, K. Odunsi, A.R. Karpf, PRAME expression and promoter hypomethylation in epithelial ovarian cancer, Oncotarget, 7 (2016) 45352-45369.

[35] K. Partheen, K. Levan, L. Osterberg, G. Horvath, Expression analysis of stage III serous ovarian adenocarcinoma distinguishes a sub-group of survivors, Eur J Cancer, 42 (2006) 2846-2854.

[36] K. Partheen, K. Levan, L. Osterberg, I. Claesson, G. Fallenius, K. Sundfeldt, G. Horvath, Four potential biomarkers as prognostic factors in stage III serous ovarian adenocarcinomas, Int J Cancer, 123 (2008) 2130-2137.

[37] K. Partheen, K. Levan, L. Osterberg, I. Claesson, K. Sundfeldt, G. Horvath, External validation suggests Integrin beta 3 as prognostic biomarker in serous ovarian adenocarcinomas, BMC Cancer, 9 (2009) 336. [38] I.G. Tkalia, L.I. Vorobyova, V.S. Svintsitsky, S.V. Nespryadko, I.V. Goncharuk, N.Y. Lukyanova, V.F. Chekhun, Clinical significance of hormonal receptor status of malignant ovarian tumors, Exp Oncol, 36 (2014) 125-133.

[39] L. Paleari, S. Gandini, N. Provinciali, M. Puntoni, N. Colombo, A. DeCensi, Clinical benefit and risk of death with endocrine therapy in ovarian cancer: A comprehensive review and meta-analysis, Gynecol Oncol, 146 (2017) 504-513.

[40] T.J. Herzog, B. Pothuri, Ovarian cancer: a focus on management of recurrent disease, Nature Clinical Practice Oncology, 3 (2006) 604.

[41] S.L. Cooke, J.D. Brenton, Evolution of platinum resistance in high-grade serous ovarian cancer, Lancet Oncol, 12 (2011) 1169-1174.

[42] R. Beckerman, C. Prives, Transcriptional regulation by p53, Cold Spring Harb Perspect Biol, 2 (2010) a000935.

[43] M. Eren, A.E. Boe, E.A. Klyachko, D.E. Vaughan, Role of plasminogen activator inhibitor-1 in senescence and aging, Semin Thromb Hemost, 40 (2014) 645-651.

[44] D.R. Green, J.E. Chipuk, p53 and metabolism: Inside the TIGAR, Cell, 126 (2006) 30-32. 
Figure 1. Unsupervised clustering of 63 samples. $\mathrm{M}$ in the color column indicates five primary cell cultures mostly including mesothelial cells. The number in the colored frames indicates the patient number. Passages of the cell lines were indicated after "\#”. Asterisks indicate the samples, which are excluded for further analyses. The close brackets indicate the samples, from which the means are calculated for further analyses.

Figure 2. Expression of determinant genes in different groups of cells. Y-axis indicates the gene expression values obtained from RNA sequencing (read counts). M, T and 8540 represent primary mesothelial cell cultures, tumor cell lines with mutant TP53 and cell line 8540 with wild-type TP53 and a KRAS mutation, respectively. Box includes all values between quartile 1 to quartile 3. By “ $T$ ", the $90^{\text {th }}$ and the $10^{\text {th }}$ percentile are shown and the outliers are indicated by dots. By "M", the individual values are shown in dots and the mean value is indicated by a bar.

Figure 3. Sensitivity of the cell lines to carboplatin. A. $\mathrm{IC}_{50}$ values obtained by in vitro chemosensitivity tests. The left cluster of cell lines are those established only once from one patient; each of the five clusters in the middle includes cell lines established from the same patient. The cluster at the right contains five primary mesothelial cell cultures. The cycle indicates the cell line with $B R C A 1$ mutation, while the dots indicate the lines with $B R C A 2$ mutations. The asterisks indicate the lines, which were derived from tumor material already exposed to carboplatin treatment. The values are shown as mean \pm SD. P indicates the patient number. $\mathrm{B}$. Comparison of the $\mathrm{IC}_{50}$ values with PFI of the corresponding patients. All patients in this selected group had no macroscopically visible residual tumor after surgery, had clinical complete remission after completing the first line treatment and had developed recurrent disease. The corresponding cell lines were derived from tumor material collected at primary 
diagnosis. The cell lines are arranged in the way with decreased $\mathrm{IC}_{50}$ values from top to bottom.

Figure 4. PRAME gene expression. HGSOC: high grade serous ovarian cancer cell lines, ordered as explained in Figure 3; M: mesothelial cell cultures; F: cell cultures containing mainly fibroblasts; OVCAR3 (NIH:OVCAR-3): an ovarian cancer cell line purchased from ATCC (ATCC HTB-161). 4A. PRAME expression in cell lines measured by RNA sequencing. 4B. Relative PRAME expression measured by RT-qPCR. 4C. PRAME expression of 66 pairs of matched primary and recurrent HGSOC tumor tissues measured by RNA sequencing. The bars present q1, median and q3; 4D. Correlation of PRAME expression in matched primary and recurrent samples. PRAME expression obtained from RNA sequencing in the 66 primary tumors are plotted against the corresponding recurrent ones. The triangles indicate two pairs of tumors both with low PRAME expression; the rhombus indicates a pair with low PRAME in recurrent tumor and higher value in primary sample; the squares indicate two pairs with low values in primary samples and high values in recurrent tumors.

Figure 5. PRAME protein expression in FFPE samples. The coding of the patients is indicated on top of each picture. The scale is indicated in the first picture on top-left with a single exception of HE staining of tumors from P8, which showed a clear HGSOC histologic type. The cell line derived from this tumor has wild-type TP53 and a KRAS mutation, which is considered to be typical for low-grade serous cancer.

Supplemental Figure S1. Morphology of the cells. Only the newly established cell lines are shown. Primary mesothelial cell cultures presented similar morphology, which is represented by P7_14433_2. 
Supplemental Figure S2. Putative p53 protein sequences predicted according to the cDNA sequencing results. The number above each block indicate the codon number of a wild-type p53 and the background colors indicate different p53 domains (transactivation domain: 1-63; proline-rich region: 64-92; DNA binding domain: 102-292; tetramerization domain: 320-355 and finally the regulatory domain at 356-393). The number of the patients is indicated at the left side. Protein changes are indicated in green, red and turquoise for deletion (“””), missense mutation and truncated protein sequences, respectively. The asterisk indicates a stop codon.

Supplementary Figure S3. Histology of the corresponding tumor of 8540. A and B: HE staining of two FFPE sections; C and D: IHC of EpCAM of the corresponding FFPE section of $\mathrm{A}$ and $\mathrm{B}$, respectively.

Supplemental Figure S4. Low-coverage sequencing plots of the newly established cell lines. The red dots indicate the individual LogR values per bin and the green lines indicate the segmented values as calculated by ASCAT v.2.0.7.

Supplementary Figure 5. Follow-up and treatment information of the patients. Patient coding and cell lines are indicated at the top of each diagram. R0 indicates no macroscopically visible residual tumor, while R1 indicates residual tumors after surgery. Blue lines indicate treatment with platinum-based drugs, while grey lines other treatment including paclitaxel, doxorubicin, topotecan, or gemcitabine. Day " 0 " indicate the time point of primary surgery. For P15 and P19, no CA125 measurement was available. The horizontal green lines indicate the threshold value of CA $125(35 \mathrm{U} / \mathrm{mL})$. 

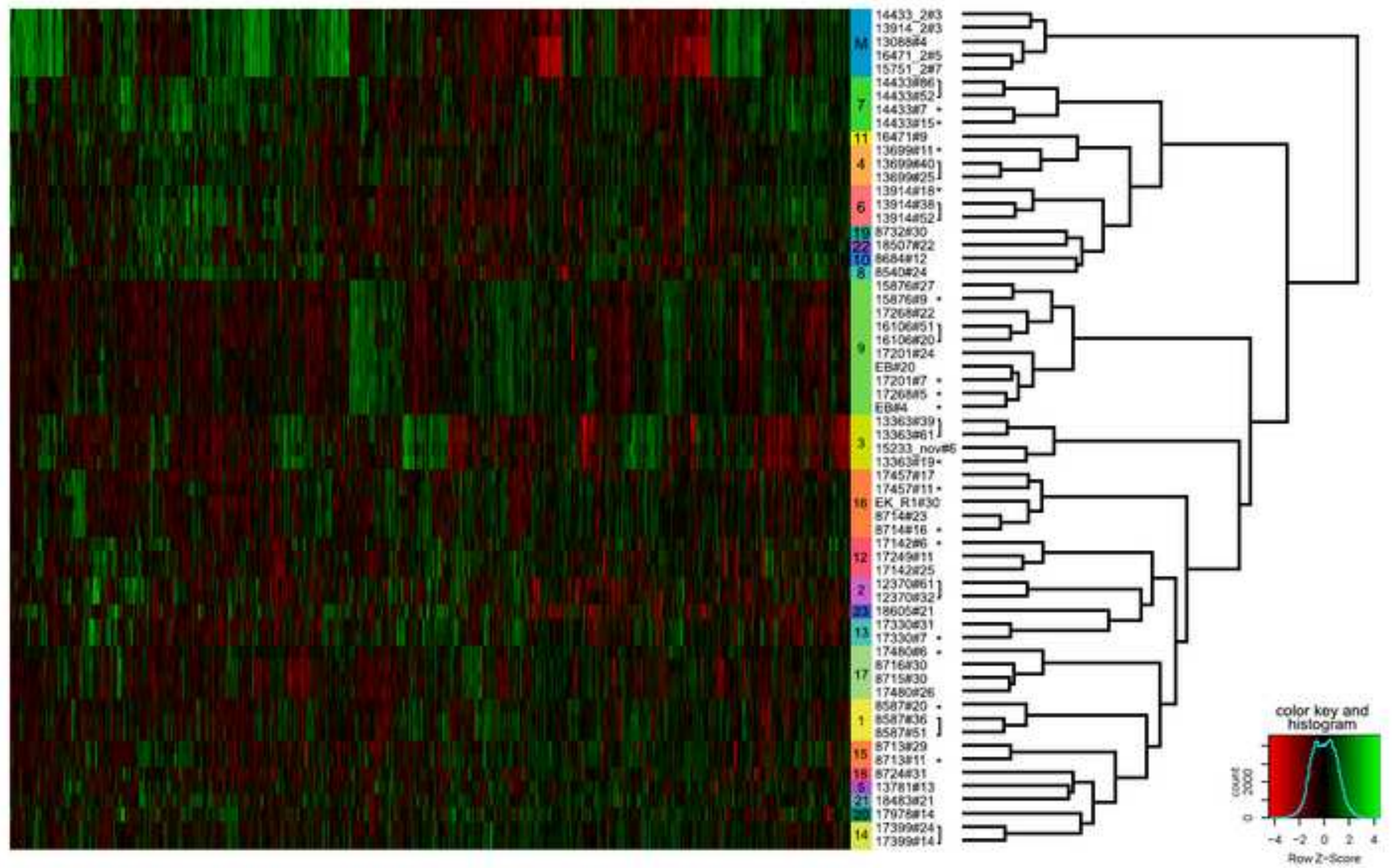
A

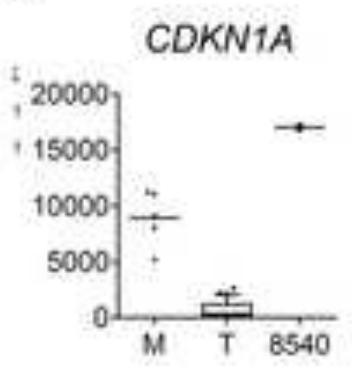

E

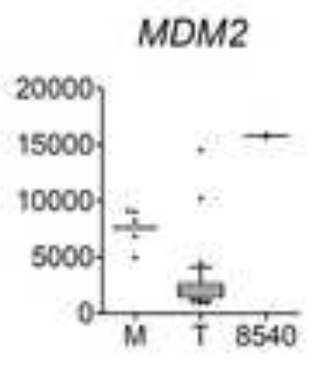

I

CCNE1

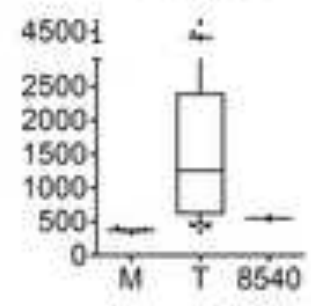

M

CCNB1

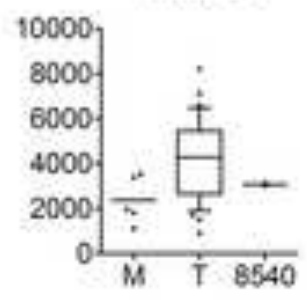

B

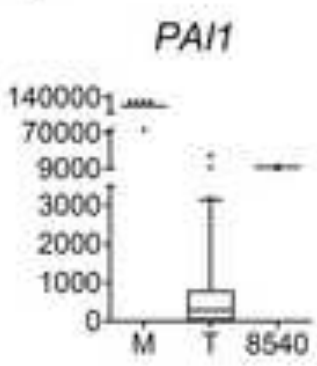

F

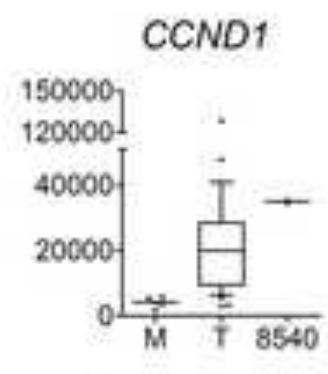

J

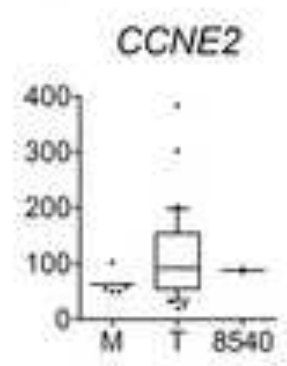

N

CCNB2

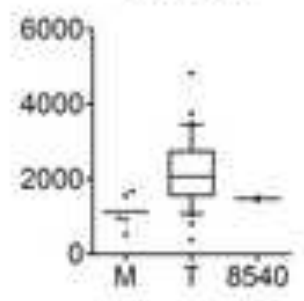

C

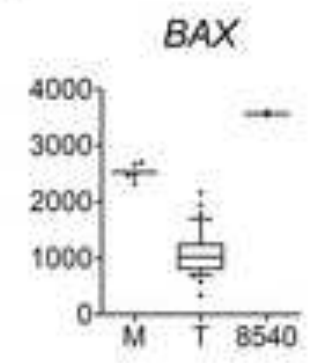

G
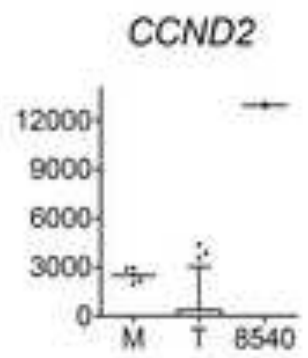

K
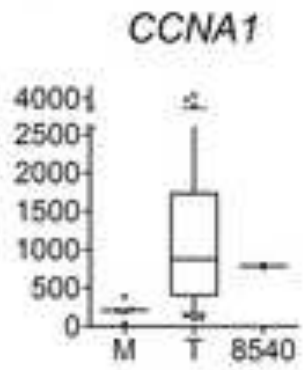

0
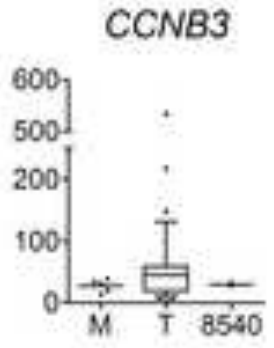

TIGAR

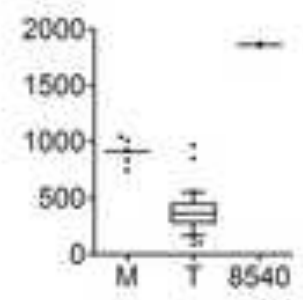

$\mathrm{H}$

CCND3

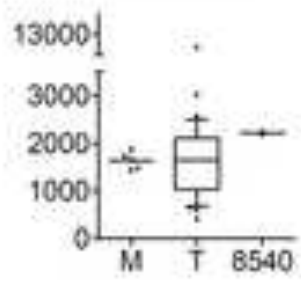

L

CCNA2

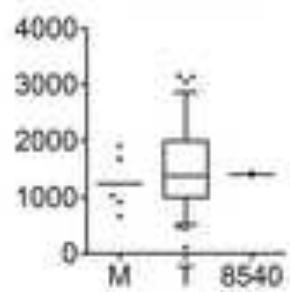

P

ESR1

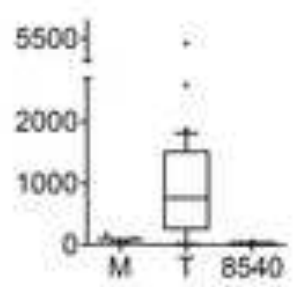




\section{gure 3}

Click here to download high resolution image

A

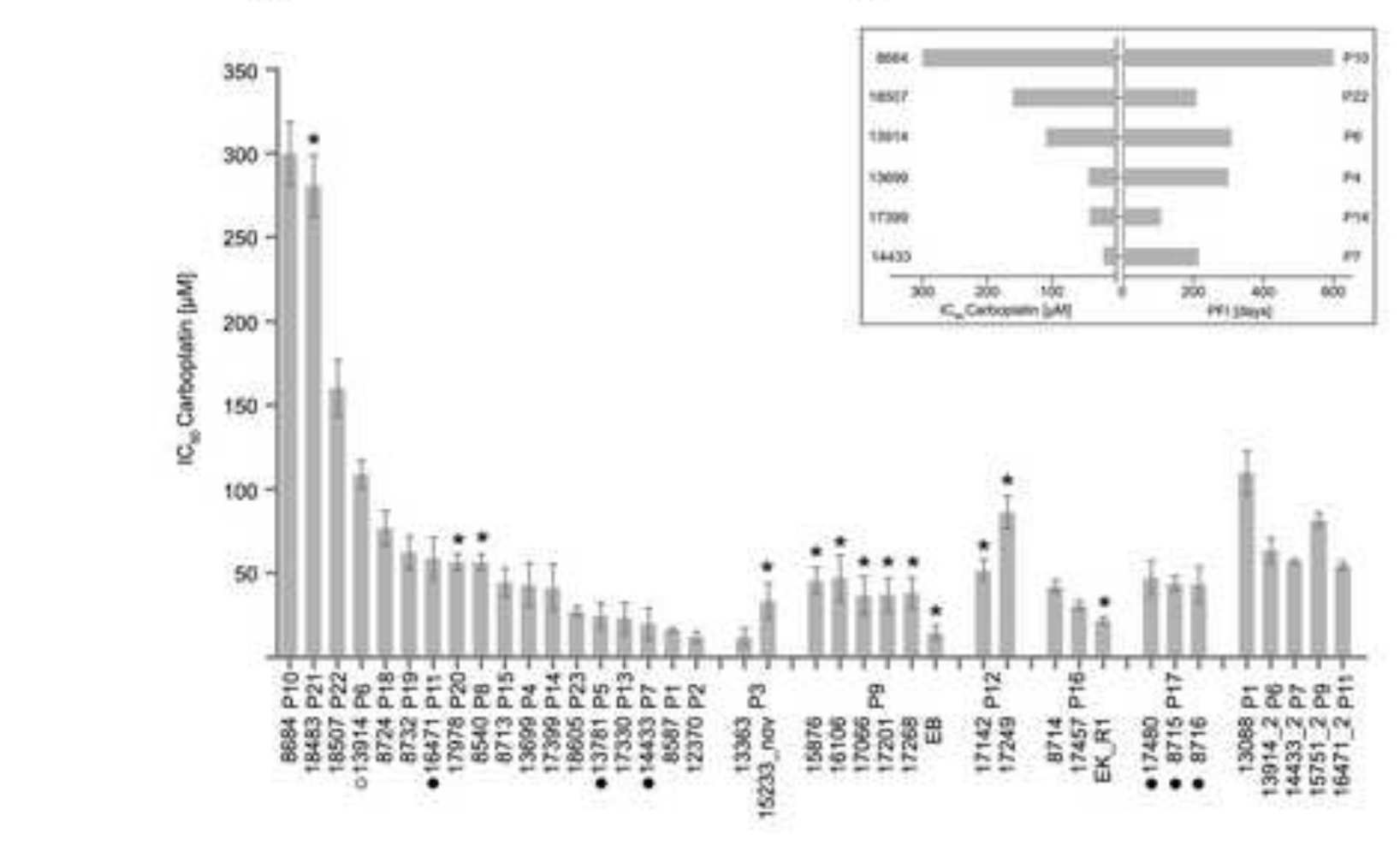

B e

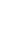


PRAME mRNA expression

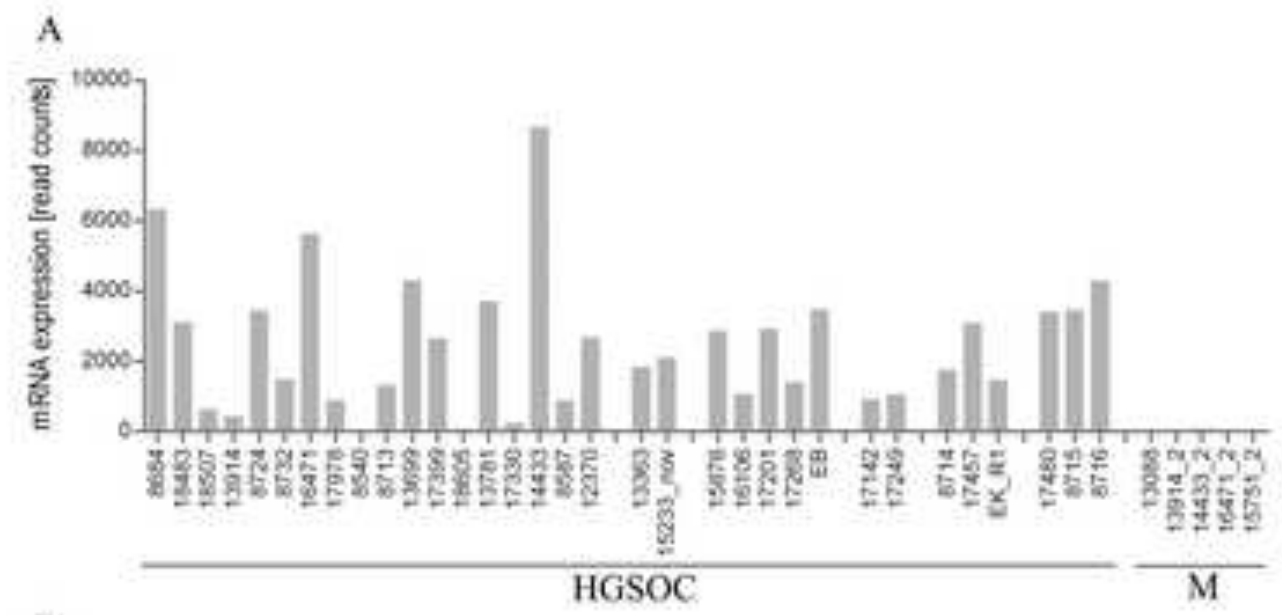

B
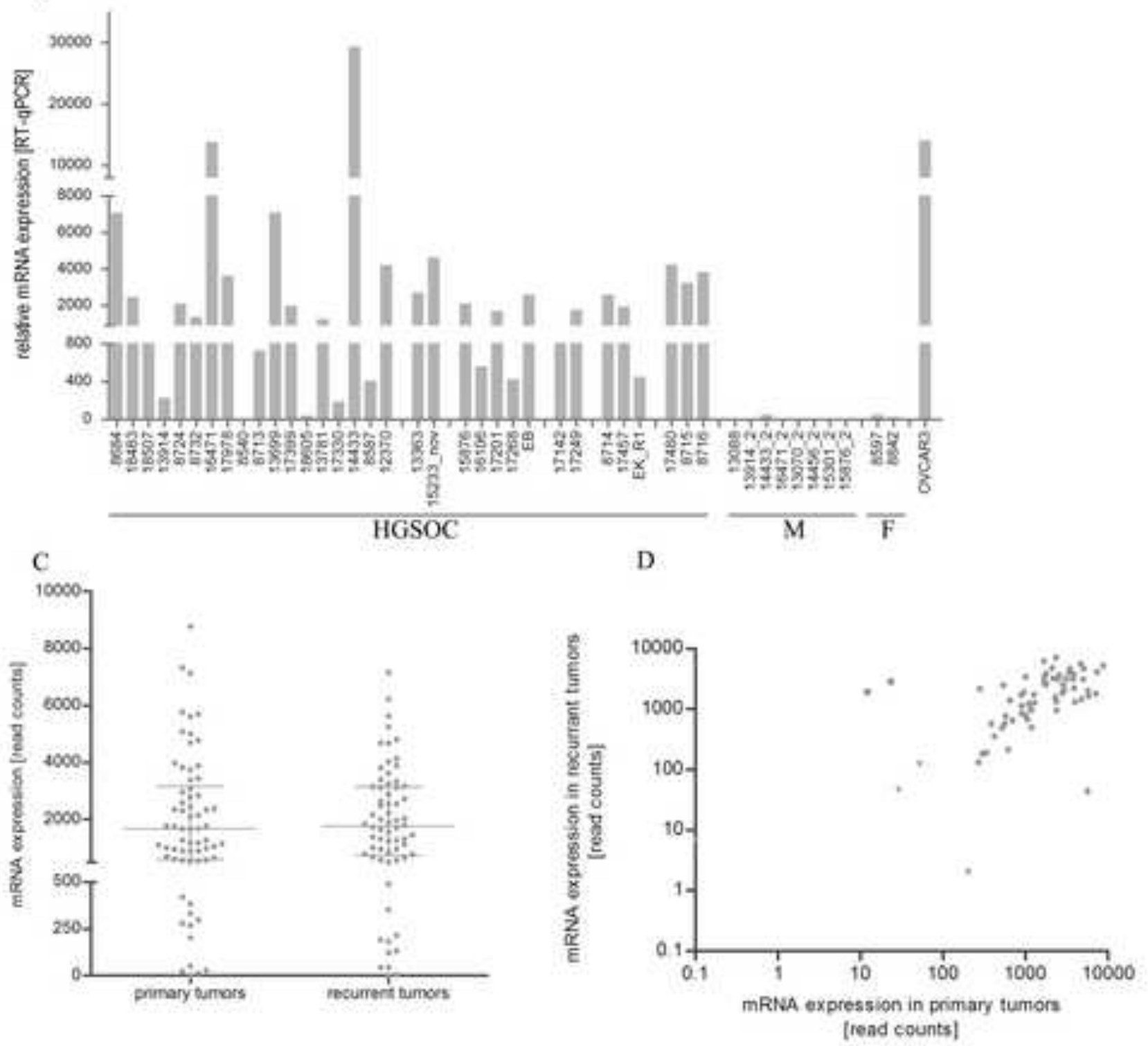
Click here to download high resolution image

P3

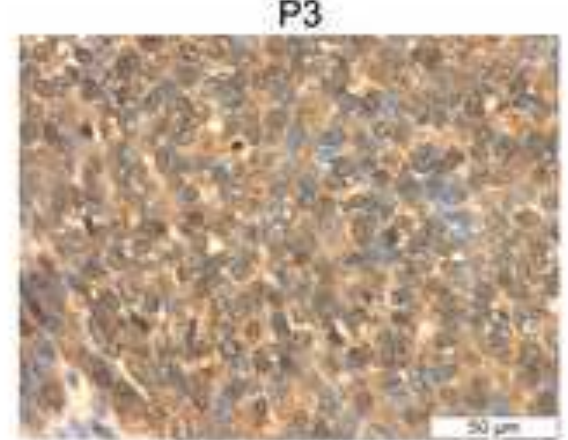

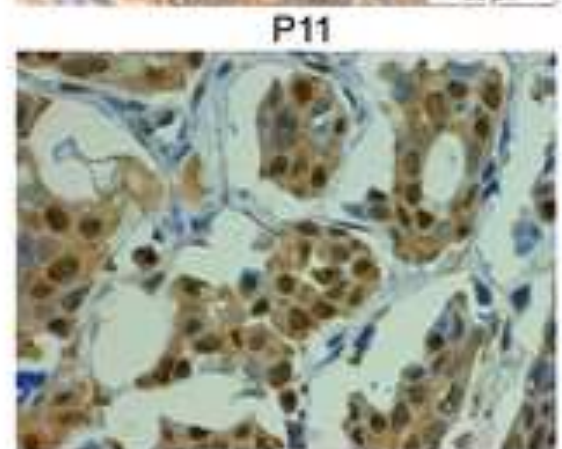
P21

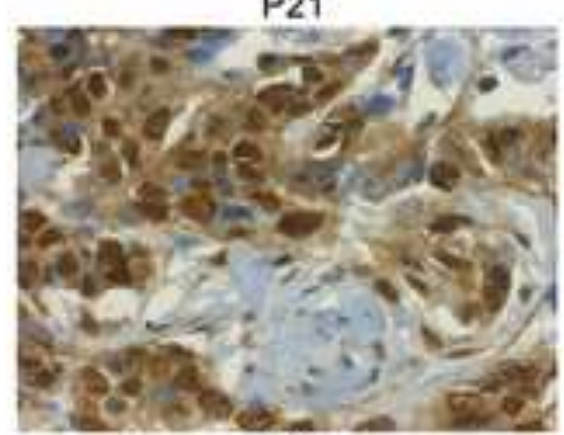

P9

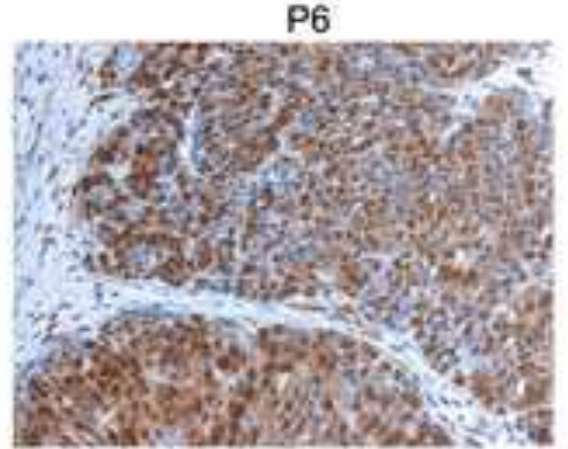

P13

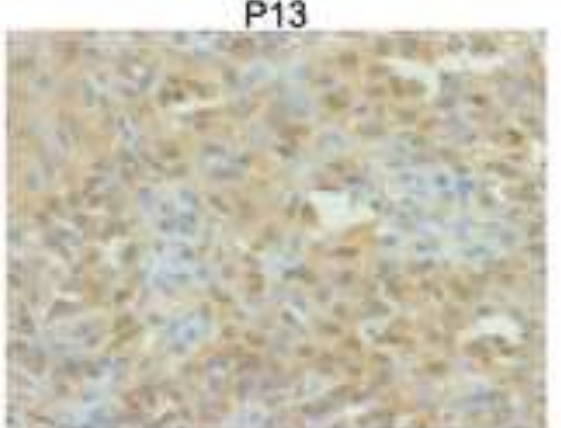

P23

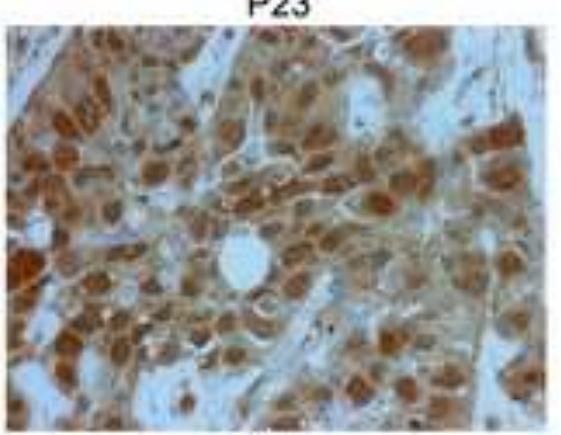

P10
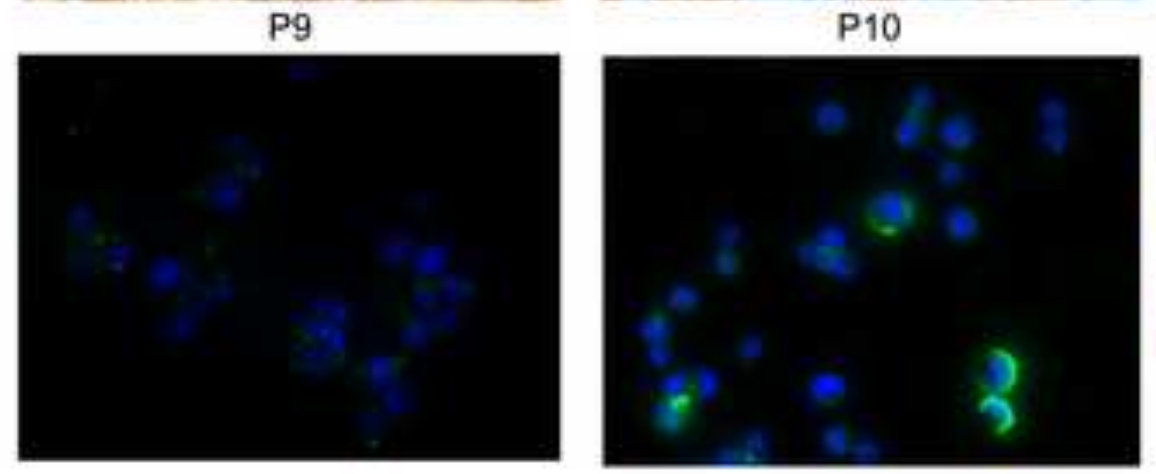

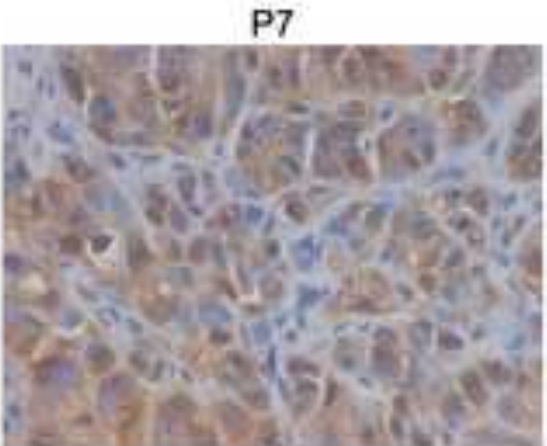

P15

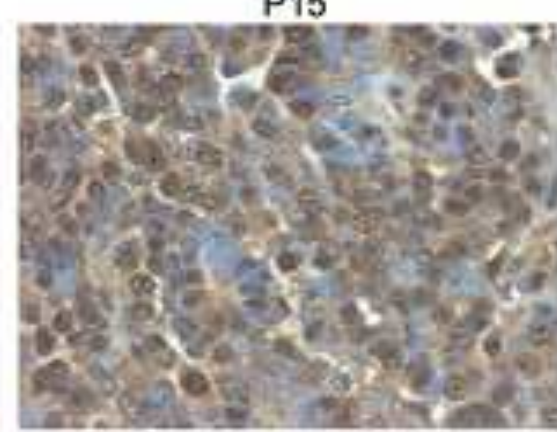

P8

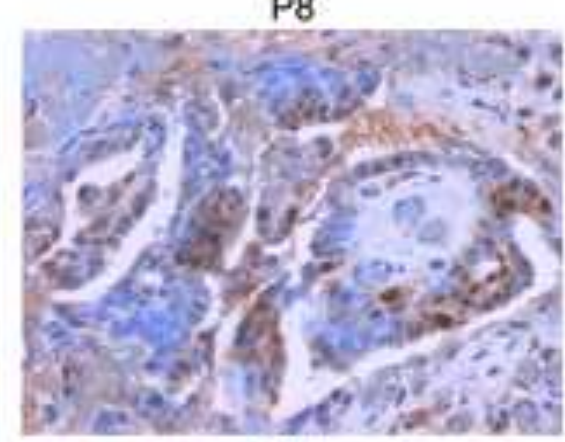

P13

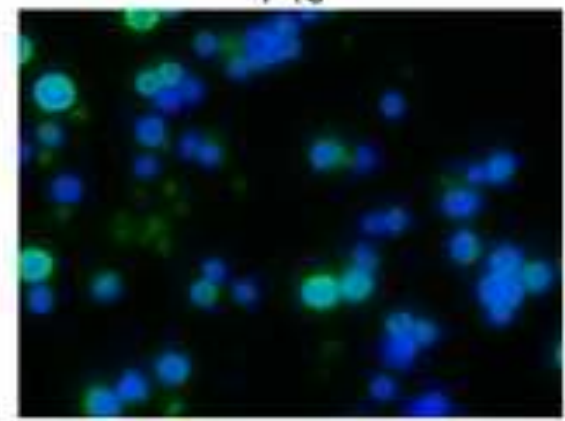

P10

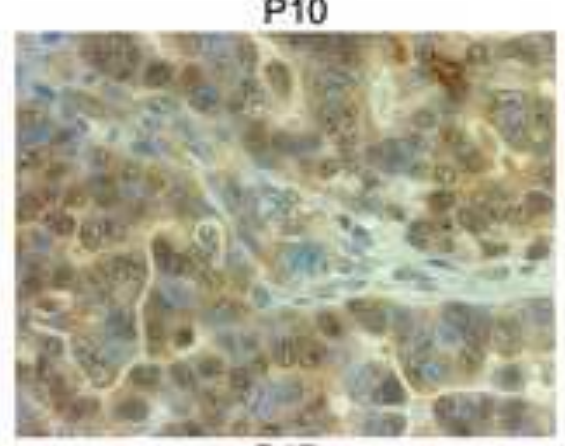
P17

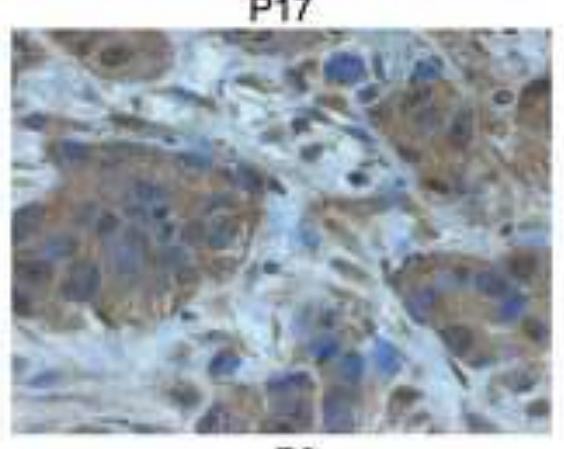

P8

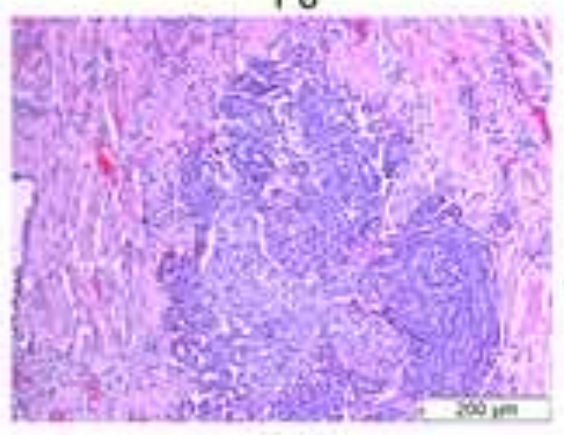

P19

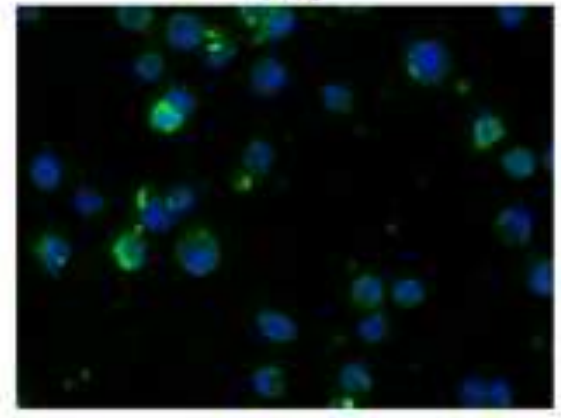


Table 1. Information on clinical data of the patients and gene mutations of the tumors

\begin{tabular}{|c|c|c|c|c|c|c|c|c|c|c|c|c|c|c|c|c|}
\hline$\frac{\stackrel{\Xi}{\Xi}}{\overline{\bar{\Xi}}}$ & 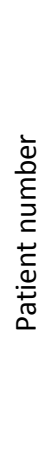 & 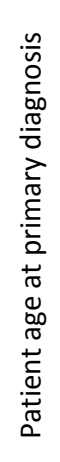 & 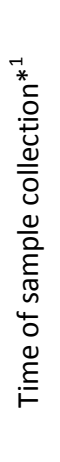 & 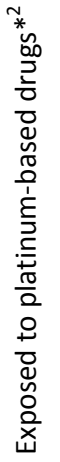 & 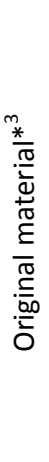 & 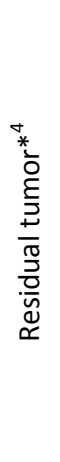 & 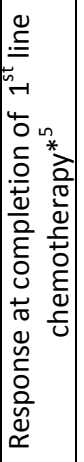 & 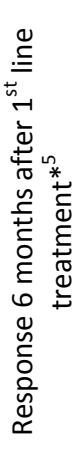 & 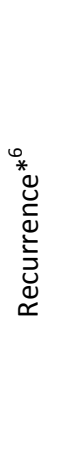 & 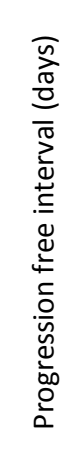 & $\begin{array}{l}\stackrel{0}{*} \\
\stackrel{*}{*} \\
\stackrel{\mathbb{N}}{\mathscr{D}} \\
0\end{array}$ & 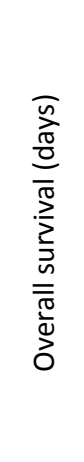 & $\begin{array}{l}\stackrel{*}{*} \\
\stackrel{n}{n} \\
\stackrel{R}{R}\end{array}$ & 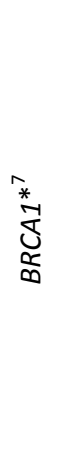 & 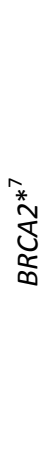 & $\underset{\frac{N}{\alpha}}{\stackrel{*}{\tilde{\alpha}}}$ \\
\hline 8587 & 1 & 49 & $P$ & 0 & $T$ & 0 & cCR & cCR & 0 & 1993 & 0 & 2119 & 1 & 0 & 0 & 0 \\
\hline 12370 & 2 & 67 & $P$ & 0 & $A$ & 1 & cPR & NA & $p$ & 0 & 1 & 275 & 1 & 0 & 0 & 0 \\
\hline 13363 & \multirow{2}{*}{3} & \multirow{2}{*}{33} & $P$ & 0 & $A$ & \multirow{2}{*}{1} & \multirow{2}{*}{ CPR } & \multirow{2}{*}{$\mathrm{cPD}$} & \multirow{2}{*}{$p$} & \multirow{2}{*}{0} & \multirow{2}{*}{1} & \multirow{2}{*}{473} & 1 & 0 & 0 & 0 \\
\hline 15233_nov & & & $R$ & 1 & $A$ & & & & & & & & 1 & 0 & 0 & 0 \\
\hline 13699 & 4 & 53 & $P$ & 0 & $A$ & 0 & CCR & $\mathrm{cCR}$ & 1 & 301 & 0 & 2059 & 1 & 0 & 0 & 0 \\
\hline 13781 & 5 & 58 & $P$ & 0 & $A$ & 1 & CCR & CPD & 1 & 107 & 1 & 887 & 1 & 0 & 1 & 0 \\
\hline 13914 & 6 & 66 & $\mathrm{P}$ & 0 & $A$ & 0 & $\mathrm{CCR}$ & NA & 1 & 309 & 1 & 880 & 1 & 1 & 0 & 0 \\
\hline 14433 & 7 & 61 & $\mathrm{P}$ & 0 & $A$ & 0 & $\mathrm{CCR}$ & $\mathrm{CPD}$ & 1 & 216 & 0 & 1727 & 1 & 0 & 1 & 0 \\
\hline 8540 & 8 & 44 & $\mathrm{PR}$ & 1 & $A$ & 1 & $\mathrm{CPR}$ & $\mathrm{CPR}$ & $p$ & 0 & 1 & 1194 & 0 & 0 & 0 & 2 \\
\hline 15876 & \multirow{6}{*}{9} & \multirow{6}{*}{79} & $P R$ & 1 & $A$ & \multirow{6}{*}{ NA } & \multirow{6}{*}{ CPR } & \multirow{6}{*}{ NA } & \multirow{6}{*}{$p$} & \multirow{6}{*}{0} & \multirow{6}{*}{1} & \multirow{6}{*}{390} & 1 & 0 & 0 & 0 \\
\hline 16106 & & & $P R$ & 1 & $A$ & & & & & & & & 1 & 0 & 0 & 0 \\
\hline 17066 & & & $\mathrm{PR}$ & 1 & $\mathrm{~A}$ & & & & & & & & 1 & 0 & 0 & 0 \\
\hline 17201 & & & $\mathrm{PR}$ & 1 & $A$ & & & & & & & & 1 & 0 & 0 & 0 \\
\hline 17268 & & & $\mathrm{PR}$ & 1 & $\mathrm{~A}$ & & & & & & & & 1 & 0 & 0 & 0 \\
\hline EB & & & $\mathrm{PR}$ & 1 & $A$ & & & & & & & & 1 & 0 & 0 & 0 \\
\hline 8684 & 10 & 68 & $\mathrm{P}$ & 0 & $T$ & 0 & $\mathrm{CCR}$ & $\mathrm{CCR}$ & 1 & 600 & 1 & 1209 & 1 & 0 & 0 & 0 \\
\hline 16471 & 11 & 58 & $\mathrm{P}$ & 0 & $A$ & 1 & $\mathrm{CCR}$ & $\mathrm{CCR}$ & 1 & 255 & 1 & 529 & 1 & 0 & 1 & 0 \\
\hline 17142 & \multirow{2}{*}{12} & \multirow{2}{*}{50} & $P R$ & 1 & $P$ & \multirow{2}{*}{0} & \multirow{2}{*}{$\mathrm{CCR}$} & \multirow{2}{*}{$\mathrm{cCR}$} & 1 & 310 & 1 & 1876 & 1 & 0 & 0 & 0 \\
\hline 17249 & & & $P R$ & 1 & $A$ & & & & 1 & 310 & 1 & $18 / 6$ & 1 & 0 & 0 & 0 \\
\hline 17330 & 13 & 68 & $\mathrm{P}$ & 0 & $\mathrm{~A}$ & 1 & $\mathrm{cCR}$ & $\mathrm{CPD}$ & 1 & 167 & 1 & 1296 & 1 & 0 & 0 & 0 \\
\hline 17399 & 14 & 72 & $\mathrm{P}$ & 0 & $\mathrm{~A}$ & 0 & cCR & CPD & 1 & 108 & 1 & 488 & 1 & 0 & 0 & 0 \\
\hline 8713 & 15 & 75 & $\mathrm{P}$ & 0 & $\mathrm{~T}$ & 1 & NA & NA & NA & NA & 0 & 56 & 1 & 0 & 2 & 0 \\
\hline 8714 & & & $\mathrm{P}$ & 0 & $\mathrm{~T}$ & & & & & & & & 1 & 0 & 0 & 0 \\
\hline 17457 & 16 & 55 & $\mathrm{P}$ & 0 & $A$ & 1 & CPR & CPD & $p$ & 0 & 1 & 466 & 1 & 0 & 0 & 0 \\
\hline EK_R1 & & & $P R$ & 1 & $\mathrm{P}$ & & & & & & & & 1 & 0 & 0 & 0 \\
\hline 17480 & & & $\mathrm{P}$ & 0 & $A$ & & & & & & & & 1 & 0 & 1 & 0 \\
\hline 8715 & 17 & 63 & $P$ & 0 & $\mathrm{~T}$ & 1 & cCR & cCR & 0 & 1166 & 0 & 1326 & 1 & 0 & 1 & 0 \\
\hline 8716 & & & $\mathrm{P}$ & 0 & $T$ & & & & & & & & 1 & 0 & 1 & 0 \\
\hline 8724 & 18 & 68 & $\mathrm{P}$ & 0 & $\mathrm{~T}$ & 1 & $\mathrm{CCR}$ & $\mathrm{CCR}$ & 1 & 223 & 1 & 674 & 1 & 0 & 2 & 0 \\
\hline 8732 & 19 & 52 & $\mathrm{P}$ & 0 & $\mathrm{~T}$ & 1 & NA & NA & NA & NA & 1 & 416 & 1 & 0 & 0 & 0 \\
\hline 17978 & 20 & 85 & $\mathrm{PR}$ & 1 & $A$ & NA & CCR & CPD & 1 & 209 & 1 & 541 & 1 & 0 & 0 & 0 \\
\hline 18483 & 21 & 54 & $\mathrm{R}$ & 1 & $A$ & 1 & CCR & CCR & 1 & 273 & 1 & 1471 & 1 & 0 & 0 & 0 \\
\hline 18507 & 22 & 76 & $P$ & 0 & $A$ & 0 & CCR & CCR & 1 & 209 & 1 & 679 & 1 & 0 & 0 & 0 \\
\hline 18605 & 23 & 65 & $P$ & 0 & $A$ & 1 & NA & NA & $p$ & 0 & 1 & 175 & 1 & 0 & 0 & 0 \\
\hline
\end{tabular}

${ }^{* 1}$ : Time of sample collection: P: at primary diagnosis; R: at recurrence; PR: at subsequent disease progression

$*^{2}$ : Exposed to platinum-based drugs: 0: no; 1 : yes

$*^{3}$ : Original material: T: tumor tissue; A: ascites; $P$ : pleural fluid

$*^{4}$ : Residual tumor: 0: no residual tumor; 1: macroscopically visible tumor; P9 and P20 did not have surgery

$*^{5}$ : Response: cCR: clinical complete response; cPR: clinical partial response; cPD: clinical progressive disease; NA: not applicable or not available

${ }^{* 6}$ : Recurrence, Death: 0: no; 1: yes; p: progressive disease; NA: not adjustable or not available; all cased of death were cancer related

$*^{7}$ : mutations in tumor cells: 0 : wild type; 1 : homozygous; 2 : heterozygous 
Table 2. Analysis of potential therapeutic targets

\begin{tabular}{|c|c|c|c|c|c|c|}
\hline 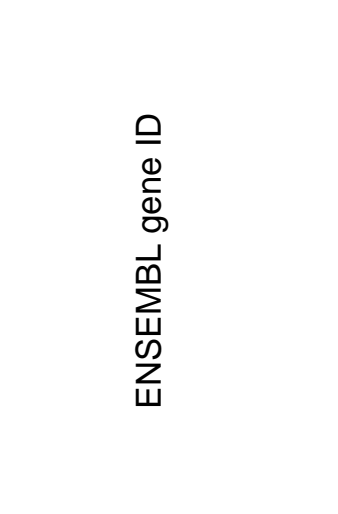 & 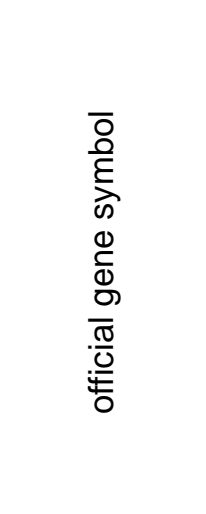 & 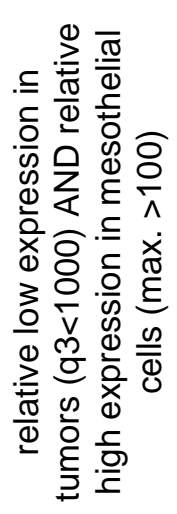 & 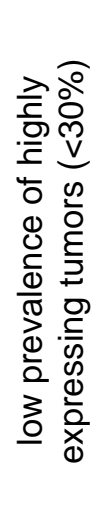 & 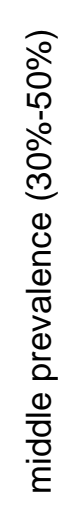 & 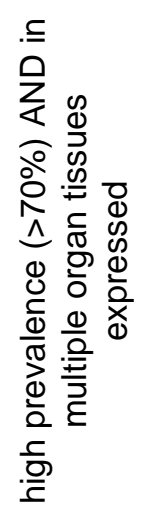 & 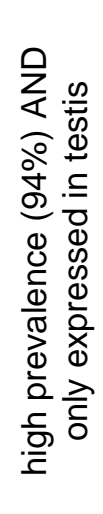 \\
\hline ENSG00000079385 & CEACAM1 & $x$ & & & & \\
\hline ENSG00000186567 & CEACAM19 & $x$ & & & & \\
\hline ENSG00000197279 & ZNF165 & $\mathrm{X}$ & & & & \\
\hline ENSG00000104327 & CALB1 & & $x$ & & & \\
\hline ENSG00000086548 & CEACAM6 & & $x$ & & & \\
\hline ENSG00000198681 & MAGEA1 & & $x$ & & & \\
\hline ENSG00000197172 & MAGEA6 & & $\mathrm{X}$ & & & \\
\hline ENSG00000124260 & MAGEA10 & & $x$ & & & \\
\hline ENSG00000046774 & MAGEC2 & & $X$ & & & \\
\hline ENSG00000117983 & MUC5B & & $\mathrm{X}$ & & & \\
\hline ENSG00000184956 & MUC6 & & $x$ & & & \\
\hline ENSG00000169550 & MUC15 & & $x$ & & & \\
\hline ENSG00000185664 & PMEL & & $\mathrm{X}$ & & & \\
\hline ENSG00000183206 & POTEC & & $x$ & & & \\
\hline ENSG00000196604 & POTEF & & $\mathrm{X}$ & & & \\
\hline ENSG00000196834 & POTEI & & $\mathrm{X}$ & & & \\
\hline ENSG00000181433 & SAGE1 & & $x$ & & & \\
\hline ENSG00000155761 & SPAG17 & & $x$ & & & \\
\hline ENSG00000241697 & TMEFF1 & & $\mathrm{X}$ & & & \\
\hline ENSG00000147381 & MAGEA4 & & & $x$ & & \\
\hline ENSG00000185247 & MAGEA11 & & & $\mathrm{X}$ & & \\
\hline ENSG00000145113 & MUC4 & & & $x$ & & \\
\hline ENSG00000104450 & SPAG1 & & & $x$ & & \\
\hline ENSG00000110195 & FOLR1 & & & & $\mathrm{X}$ & \\
\hline ENSG00000185499 & MUC1 & & & & $\mathrm{X}$ & \\
\hline ENSG00000176945 & MUC20 & & & & $x$ & \\
\hline ENSG00000185686 & PRAME & & & & & $x$ \\
\hline
\end{tabular}


Supplementary Figure S1
Click here to download Supplementary File: Kreuzinger_VonDerDecken_Supplementary Figure S1.tif

作 fo (n)

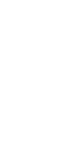
(1) (1) (1) (1) 西 . . . . . . . . . . . . 
Supplementary Figure S2
Click here to download Supplementary File: Kreuzinger_VonDerDecken_Supplementary Figure S2.pdf Click here to download Supplementary File: Kreuzinger_VonDerDecken_Supplementary Figure S2.pdf

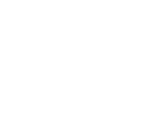

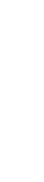
.

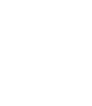
(1) (1) (1)

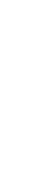

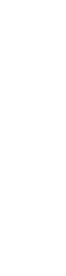
更 更 更 (1) (1)

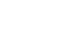

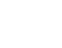

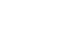

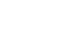

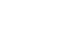

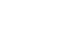

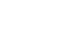

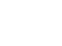

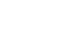

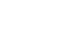

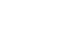

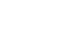

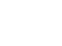




\section{Click here to download Supplementary File: Kreuzinger_VonDerDecken_Supplementary Figure S3.tif}

Supplementary Figure S3
Click here to download Su (1) (1)

cons

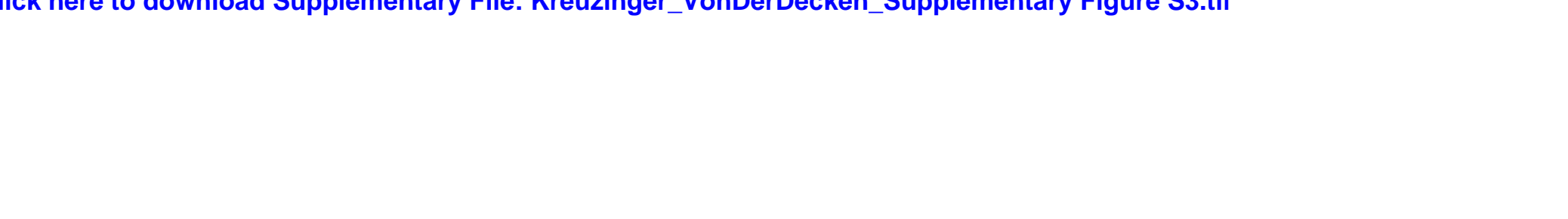

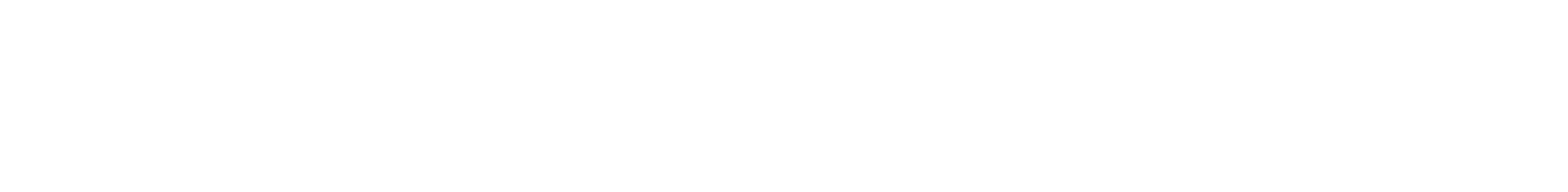


Supplementary Figure S4
Click here to download Su

Click here to download Supplementary File: Kreuzinger_VonDerDecken_Supplementary Figure S4.pdf .

\begin{abstract}
Click here to download Supplementary File:Kreuzinger_VonDerDecken_Supplementary Figure SA.pdf
(1)

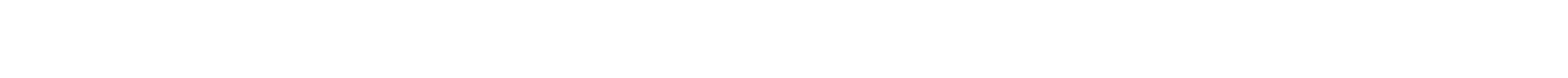
(1)

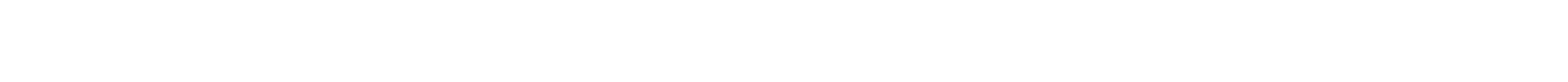

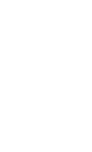

(1)

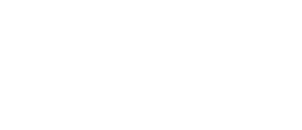

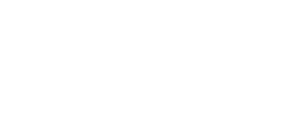
(1) . . (1) (1) . . . . 列 列 列 列 列 列 . . 列 . . 

Supplementary Table S1
Click here to download S Supplementary Table S1
Click here to download Supplementary File: Kreuzinger_VonDerDecken_Supplementary Table S1.xIsx 
Supplementary Table S2
Click here to download Supplementary File: Kreuzinger_VonDerDecken_Supplementary Table S2.docx

Supplementary Table S2
Click here to download Supplementary File: Kreuzinger_VonDerDecken_Supplementary Table S2.docx

\begin{abstract}
(a)
\end{abstract} (a)

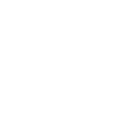

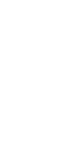

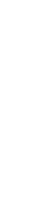
(1) (1) (1) (1) . . . . . . . . .

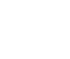


Supplementary Table S3
Click here to download Supplementary File: Kreuzinger_VonDerDecken_Supplementary Table S3.docx

\begin{abstract}
(a)
\end{abstract} (2)

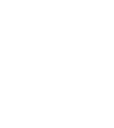

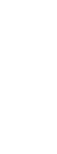

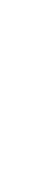

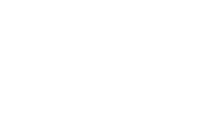

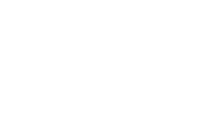

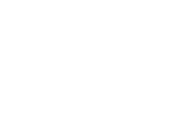

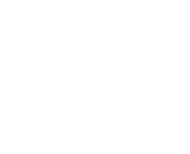

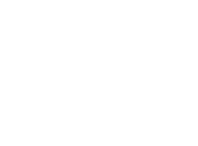

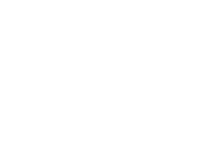
(1) (1) (1)

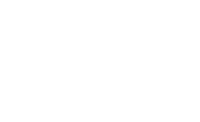


Supplementary Table S4
Click here to download Su

Click here to download Supplementary File: Kreuzinger_VonDerDecken_Supplementary Table S4.docx

(a)



Supplementary Figure S5
Click here to download Supplementary File: Kreuzinger_VonDerDecken_Supplementary Figure S5.tif

.




\section{Declaration of interest}

All authors declare that we have no financial conflict of interest that might be construed to influence the results or interpretation of the manuscript. 


\section{Title: Patient-derived cell line models revealed therapeutic targets and molecular mechanisms underlying disease progression of high grade serous ovarian cancer}

Authors: Caroline Kreuzinger ${ }^{\mathrm{a}, 1}$, Isabel von der Decken ${ }^{\mathrm{a}, 1}$, Andrea Wolf ${ }^{\mathrm{a}}$, Magdalena Gamperl ${ }^{\mathrm{a}}$, Julia Koller $^{\mathrm{a}}$, Jasmine Karacs ${ }^{\mathrm{a}}$, Stephanie Pfaffinger ${ }^{\mathrm{a}}$, Thomas Bartl ${ }^{\mathrm{a}}$, Alexander Reinthaller ${ }^{\mathrm{a}}$, Christoph Grimm $^{\mathrm{a}}$, Christian F Singer ${ }^{\mathrm{b}}$, Elena Ioana Braicu ${ }^{\mathrm{c}, \mathrm{d}}$, Paula Cunnea ${ }^{\mathrm{e}}$, Charlie Gourley ${ }^{\mathrm{f}}$, Dominiek Smeets $^{\mathrm{g}, \mathrm{h}}$, Bram Boeckx $^{\mathrm{g}, \mathrm{h}}$, Diether Lambrechts ${ }^{\mathrm{g}, \mathrm{h}}$, Paul Perco ${ }^{\mathrm{i}}$, Reinhard Horvat ${ }^{\mathrm{j}}$, Els M. J. J. Berns ${ }^{\mathrm{k}}$, Dan Cacsire Castillo-Tong ${ }^{\mathrm{a}^{*}}$

1: These authors contribute equally to the manuscript.

\section{Affiliations:}

${ }^{a}$ Translational Gynecology Group, Department of Obstetrics and Gynecology, Comprehensive Cancer Center, Medical University of Vienna, 1090 Vienna, Austria;

${ }^{\mathrm{b}}$ Department of Gynecology and Gynecologic Oncology, Gynecologic Cancer Unit, Comprehensive Cancer Center, Medical University of Vienna, 1090 Vienna, Austria;

${ }^{\mathrm{c}}$ Tumor Bank Ovarian Cancer Network, Department of Gynecology, Charité Universitätsmedizin Berlin, 13353 Berlin, Germany;

${ }^{\mathrm{d} D e p a r t m e n t ~ o f ~ G y n e c o l o g y, ~ C h a r i t e ́ ~ U n i v e r s i t a ̈ t s m e d i z i n ~ B e r l i n, ~} 13353$ Berlin, Germany;

${ }^{\mathrm{e} O v a r i a n}$ Cancer Action Research Centre, Department of Surgery and Cancer, Imperial College London, London W12 0HS, United Kingdom; 
${ }^{\mathrm{f}}$ Nicola Murray Centre for Ovarian Cancer Research, Cancer Research UK Edinburgh Centre, MRC IGMM, University of Edinburgh, Western General Hospital, Edinburgh EH4 2XR, United Kingdom;

${ }^{\mathrm{g}} \mathrm{KU}$ Leuven, Department of Human Genetics, Laboratory for Translational

Genetics, 3000 Leuven, Belgium;

${ }^{\mathrm{h}}$ VIB, VIB Center for Cancer Biology, Laboratory for Translational Genetics, 3000 Leuven, Belgium;

${ }^{\mathrm{i}}$ Emergentec Biodevelopment GmbH, 1180 Vienna, Austria; Current affiliation: Medical University Innsbruck, Department of Internal Medicine IV, 6020 Innsbruck, Austria;

${ }^{\mathrm{j}}$ Department of Clinical Pathology, Medical University of Vienna, 1090 Vienna, Austria;

${ }^{\mathrm{k}}$ Department of Medical Oncology, Erasmus MC Cancer Institute, 3000 CA Rotterdam, The Netherlands

*Correspondence: Dan Cacsire Castillo-Tong, Ebene 5Q, AKH; Waehringer Guertel 18-20, Translational Gynecology Group, Department of Obstetrics and Gynecology, Medical University of Vienna, 1090 Vienna, Austria

Email: dan.cacsire-castillo@meduniwien.ac.at 


\section{Abstract (184 words)}

High grade serous ovarian cancer (HGSOC) is the most frequent type of ovarian cancer. Most patients have primary response to platinum-based chemotherapy but frequently relapse, which leads to patient death. A lack of well documented and characterized patient-derived HGSOC cell lines is so far a major barrier to define tumor specific therapeutic targets and to study the molecular mechanisms underlying disease progression. We established 34 patient-derived HGSOC cell lines and characterized them at cellular and molecular level. Particularly, we demonstrated that a cancer-testis antigen PRAME and Estrogen Receptor could serve as therapeutic targets. Notably, data from the cell lines did not demonstrate acquired resistance due to tumor recurrence that matched with clinical observations. Finally, we presented that all HGSOC had no or very low CDKN1A (p21) expression due to loss of wild-type TP53, suggesting that loss of cell cycle control is the determinant for tumorigenesis and progression. In conclusion, patient-derived cell lines reveal that PRAME is a potential tumor specific therapeutic target in HGSOC and counteracting the down-regulation of p21 caused by loss of wild-type TP53 might be the key to impede disease progression.

Key words: acquired platinum resistance; PRAME; tumor specific antigen; TP53; $p 21$ downregulation 


\section{Introduction}

Epithelial ovarian cancer (EOC) is a rare disease with a worldwide incidence and mortality of 240000 and 150000 , respectively [1]. $75 \%$ of EOC are high grade serous ovarian cancers (HGSOC) that are often diagnosed at advanced stage. Standard of care includes debulking surgery and platinum-based therapy. Most patients show primary response; however the vast majority relapses with resistant disease, which is defined as acquired resistance. Tumor cell proliferation cannot be controlled and patients die because of disease progression. The 5-year survival rate of patients with advanced HGSOC is below 40\%. Resistance is considered the major course of the patients' death [2]. In addition, platinum-based drugs kill large numbers of nontumor cells, dramatically reducing patients' quality of life [3]. In order to improve ovarian cancer treatment, tumor specific targets have to be defined for the development of more efficient and less toxic therapies.

At the molecular level, common features of HGSOC include TP53 mutations (96\%) and loss of homologous recombination repair (HRR) due to mutational or epigenetic inactivation of the $B R C A 1 / 2$ or other HRR genes [4]. Aside from these common traits, HGSOCs are highly heterogeneous with large amounts of copy number changes and chromosomal rearrangements. The genetic instability and the loss of functional p53 and BRCA1/2 have a direct consequence of higher number of mutations in tumor cells.

To better understand the mechanisms underlying the resistance of HGSOC, well characterized experimental models representing the cellular and genetic backgrounds of HGSOC are essential. Unfortunately, by comparing copy number changes, mutations and mRNA profiles with tumors, it was discovered that the cell lines historically used for ovarian cancer research were most unlikely to be representative of HGSOC [5, 6]. For this reason, several groups including ourselves have previously attempted to establish HGSOC cell lines [6-11]. The number of the 
cell lines generated from these studies is rather low and with no systematic molecular analyses. The lack of linked clinical data about the donors is also a drawback to understand the impact of cellular and molecular characteristics on disease progression [12].

To address this challenge, we established 34 cell lines from 23 HGSOC patients and analyzed the acquired resistance in these tumors and the molecular mechanisms of disease progression. Furthermore, we investigated potential therapeutic targets using these models and validated the results using RNA sequencing data from 66 paired primary and recurrent HGSOC tumors published in a previous study [13]. The cell line models reveal that a cancer-testis antigen PRAME is a potential therapeutic target for HGSOC and the down regulation of p21 caused by the loss of wild-type TP53 is the most important event for disease progression. 


\section{Materials and methods}

2.1. Establishing and maintaining HGSOC cell lines and primary mesothelial cell culture The methods of establishing and maintaining cell lines were published previously [11]. For tumor tissues, we abandoned the previous enzymatic digestion because of a very low success rate and used a cell scraper to release the tumor cells, which were collected by centrifugation. In some ascites, many mesothelial cells grew quickly covering the surface between tumor cell clusters in the culture flasks at the beginning passages. They detached very quickly upon trypsinization ( $<2$ mins), while tumor cells needed longer to detach ( $>5 \mathrm{mins})$. Using selective trypsinization, we were able to isolate primary cell culture that predominantly contains mesothelial cells from tumor cells.

To authenticate the cell lines to patients, DNA finger printing was determined with PowerPlex 21 PCR Kit (Fitchburg, WI, USA), including 21 loci D1S1656, D2S1338, D3S1358, D5S818, D6S1043, D7S820, D8S1179, D12S391, D13S317, D16S539, D18S51, D19S433, D21S11, Amelogenin, CSF1PO, FGA, Penta D, Penta E, TH01, TPOX, and vWA [14] and compared to that of the germline DNA.

\subsection{Extraction of DNA and RNA and cDNA synthesis}

DNA and RNA were extracted with AllPrep DNA/RNA Mini Kit (QIAGEN, Venlo, The Netherlands). DNA from formalin-fixed, paraffin-embedded (FFPE) tissue and blood samples was isolated using QIAamp DNA FFPE Tissue Kit (QIAGEN) and QIAamp DNA Mini Kit (QIAGEN). The quantity was determined by measuring the absorbance at 260/280 nm with Nanodrop 1000 (Thermo Fisher Scientific, Massachusetts, USA) and the quality of RNA was controlled using Agilent RNA 6000 Nano Kit (Agilent Technologies, CA, USA) and Agilent 
2100 Bioanalyzer (Agilent Technologies). Samples with an RNA Integrity Number $>8$ were further processed for RNA sequencing. cDNA was synthesized with Omniscript RT Kit (Qiagen).

2.3. Detection of mutations in the coding region of TP53, KRAS and BRCA1/2 Alterations in the coding regions of TP53 and KRAS genes were examined by Sanger sequencing. For TP53, three DNA fragments C0 (sense: 5'-TGCTTTCCACGACGGTGAC-3'; antisense: 5'AGCAGCCTCTGGCATTCTG-3'), C1 (sense; 5'-CCTCCTCAGCATCTTATC-3', antisense: 5'-AAGAAGTGGAGAATGTCAG-3') and C2 (sense: 5'-CCAAGCAATGGATGATT-3'; antisense: 5'-TAGTGGATGGTGGTACAGTC-3') covering exon 1-4, 4-7 and 6-11, were amplified with annealing temperatures of $55^{\circ} \mathrm{C}, 50^{\circ} \mathrm{C}$ and $52^{\circ} \mathrm{C}$, respectively. For $K R A S$, one single fragment covering the whole coding region (sense: 5'-ATTTCGGACTGGGAGCGAG-3'; antisense: 5'-GCATCATCAACACCCAGATTAC-3') was amplified with an annealing temperature of $60^{\circ} \mathrm{C}$ with cDNA samples. PCR was performed with MangoMix (Bioline Reagent Ltd, London, UK) with 40 cycles of $30 \mathrm{sec}$ at $94^{\circ} \mathrm{C}, 30 \mathrm{sec}$ at the corresponding annealing temperature, and $30 \mathrm{sec}$ at $72^{\circ} \mathrm{C}$. The reactions were preceded with a 10 min denaturation step and an extension at $72^{\circ} \mathrm{C}$ for $10 \mathrm{~min}$. Mutations in the $B R C A 1$ and $B R C A 2$ coding regions were detected using TruSeq Custom Amplicon Design - AFP2, TruSeq CustomAmplicon Index Kit and Reagent Kit v2 (300cycles) and Illumina MiSeq System (Illumina; Inc., CA, USA). Once an alteration was found, it was verified with DNA or cDNA samples and confirmed in cell lines at different passages by Sanger sequencing.

2.4. Measurement of mutant DNA proportion by allele specific digital droplet PCR (ddPCR) Allele specific ddPCR systems were designed to detect the absolute copy numbers of wild type and mutant TP53 and KRAS in DNA samples. Primers and probes are shown in Supplementary Table S4. PCR was performed using ddPCR Supermix for Probes (Bio-Rad Laboratories, Inc., 
CA, USA) according the manufacturer's instruction. Signals were detected by QX200 Droplet Digital PCR System (Bio-Rad Laboratories, Inc.). We used these systems to determine the tumor cell proportion in cell culture. Additionally, we performed these assays for cell lines at higher passages to ensure that all cells harbored their specific mutations.

2.5 Low coverage whole genome sequencing and RNA-sequencing and annotation

The methods were described in detail previously [11].

\subsection{Determination of DNA copy number}

DNA copy number of TP53 and BRCA1/2 were detected with the corresponding PrimPCR ddPCR Copy Number Assays (dHsaCP1000586, dHsaCP2500367, dHsaCP2500368, respectively, Bio-Rad, CA, USA) with EIF2C1 (dHsaCP2500349) as reference gene following the manufacturer's instruction (Bio-Rad Laboratories, Inc.). DNA from a healthy individual was used as a control.

\subsection{Unsupervised clustering}

RNA sequencing data includes 63 samples from 33 cell lines with one line 17066 excluded because of bad quality of sequencing results. Euclidean distance clustering was performed on the EDA normalized data using the top $10 \%$ of the expressed genes ranked by their standard deviation across the cohort.

\subsection{Analyses of specific gene expression}

Since cell lines with low passages had higher possibility to contain other types of cells, some of the samples were excluded according to the results of unsupervised clustering (indicated in 
Figure 1). Mean expression was used for cell lines with more than one measurements at higher passages.

\subsection{In vitro carboplatin sensitivity test}

Chemo-sensitivity was measured by MTT assay using EZ4U-kit following the manufacturer's instructions (Biomedica, New Hampshire, USA). $7 \times 10^{3}$ cells per well were seeded in 96-well plates (Costar, Corning Incorporated, USA) and incubated with carboplatin (Accord Healthcare, North Harrow, UK) in triplicate at $37^{\circ} \mathrm{C}$ and $5 \% \mathrm{CO}_{2}$ for $96 \mathrm{~h}$. At least three independent experiments were performed for each cell line. $\mathrm{IC}_{50}$ values were calculated using a non-linear dose-response curve with sigmoidal fit of logarithmic mean across the triplets of each assay by GraphPad Prism 5.00 for Windows (GraphPad Software, San Diego, CA, USA; www.graphpad.com). Carboplatin concentrations ranging from $100 \mu \mathrm{g} / \mathrm{ml}(270 \mathrm{M})$ to $0.4 \mu \mathrm{g} / \mathrm{ml}$ $(1.08 \mu \mathrm{M})$ with 1:2 serial dilution between them were used in the initial test. We chose this range of concentrations, as the plasma peak value of carboplatin used for patients is around $300 \mu \mathrm{M}$. If the cells showed borderline values of sensitivity, higher or lower concentrations were included in additional tests.

\subsection{Comparison of gene expression in two different groups of cell lines}

We compared the gene expression in tumor cells established before and after exposing to carboplatin-based therapy and tumors with or without wild-type $B R C A 1 / 2$. For the latter, means were calculated in case more than one cell line was established from the same patient. The nbinomTest function of the DEseq R package was used after normalization for the identification of differentially expressed transcripts [15]. p-values were adjusted using Benjamini Hochberg methods thus controlling the false discovery rate (FDR). Transcripts with an FDR $<5 \%$, a fold- 
change value $>|5|$, and read counts $>50$ in the group showing higher read counts were considered as relevant.

\subsection{Selection of potential tumor specific antigens}

We selected genes coding for potential tumor specific antigens mainly from four sub-groups: (i) Cancer-testis like antigens (CTA); (ii) Differentiation antigens; (iii) Oncofetal antigens including $\alpha$-fetoprotein, carcinoembryonic antigen (CEA), trophoblast glycoprotein, onco-trophoblast, and solid tumor associated glycoprotein; (iv) Over-expressed antigens, mainly based on the information provided by Even-Desrumeaux et al. [16, 17]. Particularly, for CTAs, we referred the 118 genes described as "testis-selective" or "testis restricted" in the CTDatabase maintained by The Ludwig Institute for Cancer Research (http://www.cta.lncc.br/).

\subsection{Measurement of PRAME gene expression by RT-qPCR}

TaqMan Assay Gene Expression Hs01022301_m1 was used to measure the PRAME expression in cell lines according to manufacturer's instructions. A cell line with relative high expression of PRAME (according to RNA sequencing data) was used to make a dilution series to generate a standard curve. Relative expression values were calculated in fold of the standard samples. Cell line data were median-normalized for comparison of RT-qPCR results with RNA sequencing results.

\subsection{Immunohistochemistry staining and immunofluorescent staining}

Immunohistochemistry was performed as described previously [11]. Antibodies against PRAME (PA5-13679; Thermo Fischer Scientific) and EpCAM (ab32329; abcam, Cambridge, UK) was diluted at 1:400 and 1:500 in Dako Antibody-Diluent (Agilent Technologies), respectively, and incubated with the slides at $4^{\circ} \mathrm{C}$ overnight. Immunofluorescent staining (IF) was performed on 
slides pretreated with adhesion substance (Paul Marienfeld GmbH \& Co. KG, LaudaKönigshofen, Germany). 50000 cells were subjected to each well on the slides following the instruction of the manufacturer, dried and fixed in $4 \%$ paraformaldehyde. The slides were treated with $0.5 \%$ Triton X-100 in PBS, washed 3 times with PBS, each time for 3 mins and incubated with Dako Ultra Vision Block (Thermo Fischer Scientific) for 7 min at RT followed by washing in PBS-Tween 20 twice, each for $3 \mathrm{~min}$. The PRAME antibody was diluted at 1:100 in Dako Antibody-Diluent and incubated at $4^{\circ} \mathrm{C}$ overnight. Alexa Fluor 488 goat anti-Rabbit IgG (Thermo Fisher Scientific) was diluted 1:500 in Dako Antibody-Diluent and incubated for an hour at RT. DAPI staining was performed with standard protocol and the slides were mounted with Fluoromount-G (Southern Biotech, Birmingham, USA).

\subsection{Data and materials availability}

We are willing to distribute any materials (cell lines), data (RNA sequencing, low coverage sequencing, patient clinicopathological data) and protocols in the published experiments to qualified researchers for their own use upon reasonable request. 


\section{Results}

\subsection{HGSOC cell lines and patients}

34 tumor cell lines from ascites, tumor tissue and pleural fluid from 23 patients with HGSOC were established (Table 1). Part of the results regarding seven of them was reported previously [11].

From five patients (P3, P9, P12, P16, P17), we established more than one cell line from tumor material collected either from different locations or at different time points (Table 1). We also cultivated primary mesothelial cells from the ascites of eight patients, which could be passaged approximately 10 times until they underwent senescence or died. Their cell type identity was confirmed by pathologists (Supplementary Figure S1). In addition, they expressed very high level of CALB2 (>4000 read counts), which is a marker for mesothelial cells, and had little or no expression of EPCAM (26-692 read counts). This was in contrast to tumor cells, which had very high EPCAM expression (1781-47851 read counts) and no or very low CALB2 expression (0-304 read counts). Furthermore, they had wild-type TP53, while their counterpart tumor cells harbored mutant TP53 (see also 3.2).

All cell lines were authenticated to be from the corresponding patients by DNA finger printing analyses (Supplementary Table S1). The purity of the tumor cells was determined by allele specific digital droplet PCR systems measuring the proportions of tumor specific mutant TP53 or KRAS DNA copies. All cell lines have been passaged over 30 times.

\subsection{The cell lines are stable experimental models for HGSOC}

In all cell lines except one, unique TP53 alterations affecting the transcripts were detected and no wild type allele could be found (Table 1, Supplementary Table S2). The alterations in mRNA were derived either directly from DNA or from intronic changes leading to variant splicing. p53 
protein was truncated with variant C-terminals or affected by substitution or deletion of amino acids. All TP53 alterations were located in the DNA binding domain except in patients P4 and P6, whose tumors had alterations in the oligomerization domain (Supplementary Figure S2). Germline BRCA1 and BRCA2 mutations were detected in one (P6) and three (P5, P7, P11) of these cell lines, respectively, all losing wild-type alleles in tumor cells. Patient P17 presented with a somatic BRCA2 mutation with loss of heterozygosity. In addition, P15 had a heterozygous germline $B R C A 2$ mutation, which was retained in the corresponding tumor. The tumor cells from P18 harbored a heterozygous somatic BRCA2 mutation. The only TP53 wild-type cell line 8540 had a heterozygous KRAS mutation. We re-examined two FFPE blocks and confirmed the high grade serous histological type (Supplementary Figure S3). The genetic profile of the FFPE samples, the cell lines and the patient blood sample were confirmed by DNA finger printing analyses. Both mutant and wild-type DNA copies of $K R A S$ were detected in DNA samples isolated from FFPE sections by allele specific ddPCR. Sanger Sequencing confirmed that the status of TP53, BRCA1/2 and KRAS mutations was unchanged in different cell lines established from the same patients and remained stable through passaging.

Low coverage sequencing revealed that all TP53 mutant cell lines had a high incidence of copy number alterations (CNA) compared to their corresponding germline DNA, e.g. P_16099B. The TP53 wild-type and KRAS mutant cell line P8_8540 had less CNA than the TP53 mutant cell lines (Supplementary Figure S4). Nevertheless, cell lines established from the same patients presented similar pattern of CNA.

Unsupervised clustering of gene expression profiles of 63 RNA samples including cell lines with different passages (Figure 1) revealed that (i) the primary mesothelial cell cultures had different expression profiles than all tumor cell lines; (ii) different passages of the same tumor cell lines had very similar expression profiles; (iii) cell lines derived from the same patient had the most similar expression profiles, regardless of whether they were derived from different locations (e.g. 
Patient P17) or whether they were established at different time points of disease progression (e.g. Patients P3, P9, and P12).

3.3. Cell cycle pathway is the determinant for tumorigenesis and tumor progression We first examined genes, whose transcription is directly regulated by p53 and found that major genes controlling cell cycle arrest, apoptosis, survival and senescence $C D K N 1 A(p 21), B A X$, TIGAR, PAI1 and MDM2 were all down-regulated in the TP53 mutant tumor cells in comparison with the TP53 wild type cell line 8540 and the mesothelial cells (Figure 2A-E). Furthermore, cyclins controlling the cell cycle restraint points cyclin D1 (CCND1), E1 (CCNE1), A1 (CCNA1) and $B 2(C C N B 2)$ were overexpressed in all tumor cells. No obvious differences could be observed regarding the expression of other cyclins (Figure 2F-O). The KRAS mutant cell line 8540 showed additional higher expression of cyclin D2 (CCND2) (Figure 2G) in comparison to all other cell lines.

\subsection{Response to carboplatin in vitro and in vivo}

Most of the tumor cell lines showed lower $\mathrm{IC}_{50}$ values for carboplatin than the primary mesothelial cell cultures, indicating that carboplatin preferentially kills tumor cells (Figure 3A). A few cell lines showed very high values $(8684,18483)$. Different cell lines from the same patients presented different pattern of changes of the $\mathrm{IC}_{50}$ values. Some became more resistant in later established lines (e.g. 15233_nov was collected 12 months later than 13363; 17249 was collected 20 days later than 17142), while others showed the opposite change and became more sensitive (e.g. the 6 cell lines from P9 were derived from ascites isolated over a total interval of 12 months; EK_R1 was taken 6 months later then 8714 and 17457). Cell lines from P17 were established from ascites $(17480)$ and tumor tissues $(8715,8716)$ at primary diagnosis and showed similar sensitivities. 
Seven patients had no residual tumors after primary surgery, presented a clinical complete remission at the completion of the first line treatment, and then developed recurrent disease. From six of these patients, we established cell lines from primary tumor material. By comparing the $\mathrm{IC}_{50}$ value with the progression free interval (PFI), no correlation could be observed between the two parameters (Figure 3B).

We further examined clinical data of all patients (Supplementary Figure S5). 10 patients had at least one recurrence (red colored) and received at least 2 lines of platinum-based treatment. The CA-125 dropped upon the treatment of platinum-based drug by almost all cycles with a few exceptions during the whole treatment.

In addition, we did not observe particularly low $\mathrm{IC}_{50}$ values of the cell lines with $B R C A 1$ or BRCA2 mutations (Figure 3A). By comparing gene expression profiles of the cell lines from the 5 patients with only mutant $B R C A 1 / 2$ and the remaining 18 patients with at least one wild type $B R C A 1 / 2$ allele, we did not find any differentially expressed genes between the two groups, even after lowering the fold change (FC) threshold to 2.

Moreover, we compared gene expression profiles of cell lines with and without exposure to chemotherapy treatment and we found that only one gene $N B L 1$ was overexpressed in the exposed cells and eight transcripts were overexpressed in the naive cells, all being non-coding RNA genes (RP5-1114G22.2, FAM103A2P, OVAAL, RP11-527N22.1, DDX11L9, RP11438N16.1, H19, AC004540.4). By lowering the fold change (FC) threshold from 5 to 2, the results were unchanged.

3.6. PRAME and the estrogen receptor (ER) are potential therapeutic targets for HGSOC We selected a panel of tumor associated antigens including cancer-testis like antigens, differentiation antigens, oncofetal antigens and some known cancer related overexpressed antigens $[16,17]$ and examined their expression in tumor cell lines in comparison with the five 
mesothelial cell cultures. Most of the genes had no or little expression in all samples (Supplementary Table S3; AFP; BAGE2; CEACAM3, 4, 5, 7, 8, 16, 18, 20, 21; CEACAMP1, 2, 5, 7, 8, 9, 11; MAGEA3, A12, B2, C1; MUC2, 3A, 5AC, 12, 13, 19; PSG1; TEX15; TLX1, 2, 3). Some had comparable high expression in both tumor cells as well as in the mesothelial cells (e.g. EGFR, ERBB2, MGAT5, MUC16, SPAG9, TPBG, TSPYL1). 27 genes were found to be higher expressed in tumor cells than in mesothelial cells, but with different prevalence. Three had low expression in tumors and high expression in mesothelial cells and 16 had rather low prevalence of highly expressing tumors (Table 2). Four genes MAGEA4, MAGEA11, MUC4 and SPAG1 had high expression in less than $50 \%$ of the samples. Four genes had high prevalence in all tumors, among which FOLRI, MUC1 and MUC20 are expressed in multiple organs/tissues, such as kidney, salivary gland, lung, or fallopian tube (https://www.proteinatlas.org; https://www.genecards.org). PRAME was highly expressed in $94 \%$ of all tumor cell lines but not in the mesothelial cells. It was reported to be only expressed in testis tissue and to a lesser extent in ovary $[18,19]$. PRAME was not expressed in 8540 and had a very low expression in 18605 (Figure 4A). Evaluation of the RNA sequencing data with RT-qPCR confirmed that PRAME indeed had very high expression in 31/33 cell lines and was not expressed in mesothelial cells and fibroblasts (Figure 4B). By examining the RNA sequencing data from 66 matched primary and recurrent HGSOC tumor tissues from a previous study [13], high expression of PRAME was confirmed (Figure 4C) in almost all tumors. A few samples showed lower expression in both samples or in one of the matched samples (Figure 4D).

Immunohistochemistry and immunofluorescent staining of the corresponding tumor tissues showed that PRAME protein was expressed in nucleus, cytoplasm and at the membrane of the tumor cells (Figure 5).

Additionally, the gene coding for ER, ESR1 showed higher expression in TP53 mutant tumor cells and was low in 8540 and the mesothelial cells (Figure 2P). There was no or very low 
expression of the progesterone receptor $(P G R)$ in TP53 mutant tumor cells (read counts:

median=1, q1=0, q3=23) and 8540 (read count: 3$)$. 


\section{Discussion}

We successfully established 34 patient-derived HGSOC cell lines and characterized them at the cellular and molecular level. With these new cell lines, we provide useful models to the scientific community to study the tumorigenesis and progression of HGSOC. Particularly, we demonstrated that a cancer-testis antigen PRAME and the ER could serve as therapeutic targets. The experimental results did not show acquired resistance of recurrent tumors, which was in line with the clinical observations. Finally, we presented that all HGSOC had no or very low $C D K N 1 A$ (p21) expression due to loss of wild type TP53.

Since Domcke et al. [5] pointed out that many of the ovarian cancer cell lines historically used for research are unlikely to be of high grade serous origin, great efforts have been paid to establish patient-derived new models including cell lines and patient-derived xenografts. The current study presents 34 new cell line models, analyzed at the level of transcriptomics, CNV profile and mutations of TP53, BRCA1/2 and KRAS genes that are linked to clinicopathological information, treatment, and response of the patients.

Despite extensive research and the introduction of some additional therapeutic options such as Bevacizumab, ovarian cancer remains a cancer with poor prognosis [20]. Carboplatin is still the gold standard treatment for HGSOC, which kills cells nonspecifically and is highly toxic [3]. So far, identifying tumor specific targets has been a big challenge for the scientific community. Some proteins, such as the Wilms tumor protein, could not serve as an optimal target, since they are not only highly expressed in ovarian cancer cells, but also in the mesothelium [21]. An ideal therapeutic target should have a homogeneous expression in tumors and no or minimal expression in normal tissues [16]. Thus, we examined the expression levels of a panel of genes coding for possible tumor associated antigens and some membrane proteins. Four genes, MUC1, MUC20, FOLRI and PRAME with high expression levels and high prevalence across all tumor 
cell lines were identified. Among these four genes, $M U C 1$ is expressed in almost all epithelial tissues and in some hematopoietic cells [22] and MUC20 was detected in multiple organs, such as kidney, lung, liver, and the digestion system [23]. Expression of FOLRI was also found in normal tissues such as kidney, bladder, salivary glands, lung and some cells of the nerve system [24]. In contrast to the multiple tissue expression of these three genes, PRAME expression is only found in normal testis tissues and to a lesser extent in the ovary. Since the ovaries of the HGSOC patients were affected by tumors and are usually removed during surgery, targeting PRAME would not affect normal tissues. Our results also showed that $P R A M E$ was not expressed in mesothelial cells lining the peritoneal cavity and in fibroblasts, which makes PRAME an optimal target for therapy development.

PRAME, the preferentially expressed antigen in melanoma gene, was first detected in a mRNA study of human testis [25]. It codes a membrane-bound protein and triggers autologous cytotoxic T cell-mediated immune responses, presumably through retinoic acid signaling [18, 26, 27]. Its overexpression in several solid tumors, such as melanoma [28], breast cancer [19], and sarcomas $[29,30]$ as well as in hematological malignancies [27] makes it an interesting therapeutic target. Recent studies using recombinant PRAME and an immunostimulant demonstrated a cellular immune response in patients in addition to safety [31, 32]. Furthermore, a polyclonal antibody was produced against PRAME and was shown to bind recombinant PRAME ex vivo as well as different cells in vitro [33]. In ovarian cancer, PRAME was found to be frequently expressed in epithelial cancer at both mRNA and protein levels, which was regulated by DNA methylation [34], and to have prognostic value for stage III serous cancer [35-37] . Further investigation will be needed to understand the expression activation and functions of PRAME in TP53 mutant HGSOC to provide the basis for drug development, for which the new patient-derived cell lines presented in this study are ideal models. 
Patients with serous ovarian cancer that have positive hormonal receptor status were shown to have worse survival than those with negative receptor status [38]. Since the ovary produces estrogen, which could promote cell growth upon binding to its receptor ER, we also examined the expression level of ESRl gene and found a high expression of this gene in most of the TP53 mutant tumor cell lines. Endocrine therapy was shown to bring benefits to patients with advanced epithelial ovarian cancer [39], probably by blocking the ESR1 promoted tumor cell growth. Even though the effect was not specified to HGSOC, it is worth further investigation in a well-defined population. Using our cell line models, the transcriptional regulation of ESR1 could be further studied.

Originally, platinum resistance was defined for tumors, which recur within 6 months after completing first line therapy [40]. This concept of clinical resistance, however, does not necessarily associate with the response of tumor cells to drug. Other possible reasons for a rapid recurrence of tumors could be the quick exponential proliferation or/and higher number of living tumor cells after therapy due to poor accessibility of the drug. This clinical definition of resistance was equaled to a cellular resistance in 2011 for ovarian cancer [41], while the hypotheses of acquired resistance and the evolution of resistance were proposed. Our cell line models showed that recurrent tumor cells did not become more "resistant" to carboplatin than their primary counterparts, which was in accordance with patient response presented by clinical data, suggesting that recurrent tumors resemble their primary ancestors. Molecular analyses of their transcriptional profiling and $\mathrm{CNV}$ profiles further suggest the similarities of the primary and recurrent tumor cells. Nevertheless, the current study included a small cohort of 23 patients and establishing cell lines might involve selections of tumors with distinct molecular characteristics. Thus, evaluation in a larger cohort of patients will be needed to clarify this issue. By examining genes, whose transcription is directly regulated by p53 [42], a clear down regulation was observed for the key genes, which regulate the cell cycle (p21, CNKN1A), 
apoptosis $(B A X)$, senescence (PAI1) [43] and survival (TIGAR) [44] in all TP53 mutant cell lines, indicating that the loss of wild-type p53 functions is the major driving force of tumor cell progression. We also observed higher expression of genes coding for cyclins D1 (CCND1), E1 (CCNE1), A1 (CCNA1) and B2 (CCNB2), demonstrating that TP53 mutant tumor cells had a high endogenous level of all cyclins needed to pass the G1/S and G2/M restriction points. Synchronized with the down-regulation of $\mathrm{p} 21$, the inhibitor of the cyclin and the cyclindependent kinase complexes, tumor cells lost the restraints in their cell cycle and were directed to proliferation. Our data suggest that loss of the wild-type TP53 is not only the reason for tumorigenesis but also the driver of tumor progression. Although well established, we confirmed in the current study that all these cellular processes are common for all TP53 mutant HGSOC. It is important to emphasize on the determinant role of cell cycle control in HGSOC, so that efforts could be directed towards the main reason of disease progression in the development of new therapies.

Taken together, we (i) established and molecularly analyzed 34 cell lines from 23 HGSOC patients, (ii) showed that loss of TP53 wild type was the main driving force of tumorigenesis and tumor progression, and (iii) identified $P R A M E$ as a potential therapeutic target. The cell line models can be applied for therapy development in addition to the investigation of molecular mechanisms in disease progression.

\section{Acknowledgements}

This work was supported by European Community’s Seventh Framework Programme under grant agreement No. 279113-2 (OCTIPS; www.octips.eu). All authors declare that we have no financial conflict of interest that might be construed to influence the results or interpretation of the manuscript. 


\section{Author contributions}

Conceptualization, C.K., I.V.D.D., E.I.B., P.C., C.G., D.L., E.M.B, and D.C.C-T; Methodology, C.K., I.V.D.D., A.W., M.G., JA.K., JU.K., S.P., A.R., C.G., C.F.S., D.S., R.H., B.B., and P.P.; Formal Analysis, B.B. and P.P.; Investigation, C.K., I.V.D.D., A.W., M.G., JA.K., JU.K., S.P. and D.C.C-T; Resources, A.R., C.G., T.B. and R. H.; Data curation, C.K., I.V.D.D., A.W., M.G., JA.K., JU.K., S.P., D.L., D.S., and B.B; Writing - original Draft C.K., I.V.D.D. and D.C.C-T; Writing - Review \& Editing, C.K., I.V.D.D., C.G., E.I.B., P.C., C.G., D.S., E.M.B. and D.C.C-T; Visualization, I.V.D.D. and B.B.; Project Administration and Supervision, D.C.C-T; Funding Acquisition, E.I.B., C.G., D.L., E.M.B. and D.C.C.-T. 


\section{References}

[1] D.D. Bowtell, S. Bohm, A.A. Ahmed, P.J. Aspuria, R.C. Bast, Jr., V. Beral, J.S. Berek, M.J. Birrer, S. Blagden, M.A. Bookman, J.D. Brenton, K.B. Chiappinelli, F.C. Martins, G. Coukos, R. Drapkin, R. Edmondson, C. Fotopoulou, H. Gabra, J. Galon, C. Gourley, V. Heong, D.G. Huntsman, M. Iwanicki, B.Y. Karlan, A. Kaye, E. Lengyel, D.A. Levine, K.H. Lu, I.A. McNeish, U. Menon, S.A. Narod, B.H. Nelson, K.P. Nephew, P. Pharoah, D.J. Powell, Jr., P. Ramos, I.L. Romero, C.L. Scott, A.K. Sood, E.A. Stronach, F.R. Balkwill, Rethinking ovarian cancer II: reducing mortality from high-grade serous ovarian cancer, Nat Rev Cancer, 15 (2015) 668-679.

[2] E.M. Berns, D.D. Bowtell, The changing view of high-grade serous ovarian cancer, Cancer Res, 72 (2012) 2701-2704.

[3] M.G. Apps, E.H. Choi, N.J. Wheate, The state-of-play and future of platinum drugs, Endocr Relat Cancer, 22 (2015) R219-233.

[4] N. Cancer Genome Atlas Research, Integrated genomic analyses of ovarian carcinoma, Nature, 474 (2011) 609-615.

[5] S. Domcke, R. Sinha, D.A. Levine, C. Sander, N. Schultz, Evaluating cell lines as tumour models by comparison of genomic profiles, Nat Commun, 4 (2013) 2126.

[6] M.S. Anglesio, K.C. Wiegand, N. Melnyk, C. Chow, C. Salamanca, L.M. Prentice, J. Senz, W. Yang, M.A. Spillman, D.R. Cochrane, K. Shumansky, S.P. Shah, S.E. Kalloger, D.G. Huntsman, Type-specific cell line models for type-specific ovarian cancer research, PLoS One, 8 (2013) e72162.

[7] I.J. Letourneau, M.C. Quinn, L.L. Wang, L. Portelance, K.Y. Caceres, L. Cyr, N. Delvoye, L. Meunier, M. de Ladurantaye, Z. Shen, S.L. Arcand, P.N. Tonin, D.M. Provencher, A.M. Mes-Masson, Derivation and characterization of matched cell lines from primary and recurrent serous ovarian cancer, BMC Cancer, 12 (2012) 379.

[8] Z. Pan, J. Hooley, D.H. Smith, P. Young, P.E. Roberts, J.P. Mather, Establishment of human ovarian serous carcinomas cell lines in serum free media, Methods, 56 (2012) 432-439.

[9] T. Sueblinvong, R. Ghebre, Y. lizuka, S.E. Pambuccian, R. Isaksson Vogel, A.P. Skubitz, M. Bazzaro, Establishment, characterization and downstream application of primary ovarian cancer cells derived from solid tumors, PLoS One, 7 (2012) e50519.

[10] T.A. Ince, A.D. Sousa, M.A. Jones, J.C. Harrell, E.S. Agoston, M. Krohn, L.M. Selfors, W. Liu, K. Chen, M. Yong, P. Buchwald, B. Wang, K.S. Hale, E. Cohick, P. Sergent, A. Witt, Z. Kozhekbaeva, S. Gao, A.T. Agoston, M.A. Merritt, R. Foster, B.R. Rueda, C.P. Crum, J.S. Brugge, G.B. Mills, Characterization of twenty-five ovarian tumour cell lines that phenocopy primary tumours, Nat Commun, 6 (2015) 7419. [11] C. Kreuzinger, M. Gamperl, A. Wolf, G. Heinze, A. Geroldinger, D. Lambrechts, B. Boeckx, D. Smeets, R. Horvat, S. Aust, G. Hamilton, R. Zeillinger, D. Cacsire Castillo-Tong, Molecular characterization of 7 new established cell lines from high grade serous ovarian cancer, Cancer Lett, 362 (2015) 218-228.

[12] F. Jacob, S. Nixdorf, N.F. Hacker, V.A. Heinzelmann-Schwarz, Reliable in vitro studies require appropriate ovarian cancer cell lines, J Ovarian Res, 7 (2014) 60.

[13] C. Kreuzinger, A. Geroldinger, D. Smeets, E.I. Braicu, J. Sehouli, J. Koller, A. Wolf, S. Darb-Esfahani, K. Joehrens, I. Vergote, A. Vanderstichele, B. Boeckx, D. Lambrechts, H. Gabra, G.B.A. Wisman, F. Trillsch, G. Heinze, R. Horvat, S. Polterauer, E. Berns, C. Theillet, D. Cacsire Castillo-Tong, A Complex Network of Tumor Microenvironment in Human High-Grade Serous Ovarian Cancer, Clin Cancer Res, 23 (2017) 76217632.

[14] M.G. Ensenberger, C.R. Hill, R.S. McLaren, C.J. Sprecher, D.R. Storts, Developmental validation of the PowerPlex((R)) 21 System, Forensic Sci Int Genet, 9 (2014) 169-178.

[15] S. Anders, W. Huber, Differential expression analysis for sequence count data, Genome Biol, 11 (2010) R106.

[16] K. Even-Desrumeaux, D. Baty, P. Chames, State of the art in tumor antigen and biomarker discovery, Cancers (Basel), 3 (2011) 2554-2596. 
[17] N. Beauchemin, A. Arabzadeh, Carcinoembryonic antigen-related cell adhesion molecules (CEACAMs) in cancer progression and metastasis, Cancer Metastasis Rev, 32 (2013) 643-671.

[18] H. Ikeda, B. Lethe, F. Lehmann, N. van Baren, J.F. Baurain, C. de Smet, H. Chambost, M. Vitale, A. Moretta, T. Boon, P.G. Coulie, Characterization of an antigen that is recognized on a melanoma showing partial HLA loss by CTL expressing an NK inhibitory receptor, Immunity, 6 (1997) 199-208.

[19] M.T. Epping, A.A. Hart, A.M. Glas, O. Krijgsman, R. Bernards, PRAME expression and clinical outcome of breast cancer, Br J Cancer, 99 (2008) 398-403.

[20] S. Mahner, L. Woelber, V. Mueller, I. Witzel, K. Prieske, D. Grimm, V.A.G. Keller, F. Trillsch, Beyond Bevacizumab: An Outlook to New Anti-Angiogenics for the Treatment of Ovarian Cancer, Front Oncol, 5 (2015) 211.

[21] C. Barcena, E. Oliva, WT1 expression in the female genital tract, Adv Anat Pathol, 18 (2011) 454-465. [22] A.M. Sousa, P.M. Grandgenett, L. David, R. Almeida, M.A. Hollingsworth, F. Santos-Silva, Reflections on MUC1 glycoprotein: the hidden potential of isoforms in carcinogenesis, APMIS, 124 (2016) 913-924. [23] T. Higuchi, T. Orita, S. Nakanishi, K. Katsuya, H. Watanabe, Y. Yamasaki, I. Waga, T. Nanayama, Y. Yamamoto, W. Munger, H.W. Sun, R.J. Falk, J.C. Jennette, D.A. Alcorta, H. Li, T. Yamamoto, Y. Saito, M. Nakamura, Molecular cloning, genomic structure, and expression analysis of MUC20, a novel mucin protein, up-regulated in injured kidney, J Biol Chem, 279 (2004) 1968-1979.

[24] L.E. Kelemen, The role of folate receptor alpha in cancer development, progression and treatment: cause, consequence or innocent bystander?, Int J Cancer, 119 (2006) 243-250.

[25] A. Pawlak, C. Toussaint, I. Levy, F. Bulle, M. Poyard, R. Barouki, G. Guellaen, Characterization of a large population of mRNAs from human testis, Genomics, 26 (1995) 151-158.

[26] N. van Baren, H. Chambost, A. Ferrant, L. Michaux, H. Ikeda, I. Millard, D. Olive, T. Boon, P.G. Coulie, PRAME, a gene encoding an antigen recognized on a human melanoma by cytolytic $T$ cells, is expressed in acute leukaemia cells, Br J Haematol, 102 (1998) 1376-1379.

[27] F. Wadelin, J. Fulton, P.A. McEwan, K.A. Spriggs, J. Emsley, D.M. Heery, Leucine-rich repeat protein PRAME: expression, potential functions and clinical implications for leukaemia, Mol Cancer, 9 (2010) 226. [28] M.G. Field, C.L. Decatur, S. Kurtenbach, G. Gezgin, P.A. van der Velden, M.J. Jager, K.N. Kozak, J.W. Harbour, PRAME as an Independent Biomarker for Metastasis in Uveal Melanoma, Clin Cancer Res, 22 (2016) 1234-1242.

[29] S.M. Pollack, Y. Li, M.J. Blaisdell, E.A. Farrar, J. Chou, B.L. Hoch, E.T. Loggers, E. Rodler, J.F. Eary, E.U. Conrad, 3rd, R.L. Jones, C. Yee, NYESO-1/LAGE-1s and PRAME are targets for antigen specific T cells in chondrosarcoma following treatment with 5-Aza-2-deoxycitabine, PLoS One, 7 (2012) e32165.

[30] P. Tan, C. Zou, B. Yong, J. Han, L. Zhang, Q. Su, J. Yin, J. Wang, G. Huang, T. Peng, J. Shen, Expression and prognostic relevance of PRAME in primary osteosarcoma, Biochem Biophys Res Commun, 419 (2012) 801-808.

[31] J.L. Pujol, T. De Pas, A. Rittmeyer, E. Vallieres, B. Kubisa, E. Levchenko, S. Wiesemann, G.A. Masters, R. Shen, S.A. Tjulandin, H.S. Hofmann, N. Vanhoutte, B. Salaun, M. Debois, S. Jarnjak, P.M. De Sousa Alves, J. Louahed, V.G. Brichard, F.F. Lehmann, Safety and Immunogenicity of the PRAME Cancer Immunotherapeutic in Patients with Resected Non-Small Cell Lung Cancer: A Phase I Dose Escalation Study, J Thorac Oncol, 11 (2016) 2208-2217.

[32] R. Gutzmer, L. Rivoltini, E. Levchenko, A. Testori, J. Utikal, P.A. Ascierto, L. Demidov, J.J. Grob, R. Ridolfi, D. Schadendorf, P. Queirolo, A. Santoro, C. Loquai, B. Dreno, A. Hauschild, E. Schultz, T.P. Lesimple, N. Vanhoutte, B. Salaun, M. Gillet, S. Jarnjak, P.M. De Sousa Alves, J. Louahed, V.G. Brichard, F.F. Lehmann, Safety and immunogenicity of the PRAME cancer immunotherapeutic in metastatic melanoma: results of a phase I dose escalation study, ESMO Open, 1 (2016) e000068.

[33] D. Pankov, L. Sjostrom, T. Kalidindi, S.G. Lee, K. Sjostrom, R. Gardner, M.R. McDevitt, R. O'Reilly, D.L.J. Thorek, S.M. Larson, D. Veach, D. Ulmert, In vivo immuno-targeting of an extracellular epitope of membrane bound preferentially expressed antigen in melanoma (PRAME), Oncotarget, 8 (2017) 6591765931. 
[34] W. Zhang, C.J. Barger, K.H. Eng, D. Klinkebiel, P.A. Link, A. Omilian, W. Bshara, K. Odunsi, A.R. Karpf, PRAME expression and promoter hypomethylation in epithelial ovarian cancer, Oncotarget, 7 (2016) 45352-45369.

[35] K. Partheen, K. Levan, L. Osterberg, G. Horvath, Expression analysis of stage III serous ovarian adenocarcinoma distinguishes a sub-group of survivors, Eur J Cancer, 42 (2006) 2846-2854.

[36] K. Partheen, K. Levan, L. Osterberg, I. Claesson, G. Fallenius, K. Sundfeldt, G. Horvath, Four potential biomarkers as prognostic factors in stage III serous ovarian adenocarcinomas, Int J Cancer, 123 (2008) 2130-2137.

[37] K. Partheen, K. Levan, L. Osterberg, I. Claesson, K. Sundfeldt, G. Horvath, External validation suggests Integrin beta 3 as prognostic biomarker in serous ovarian adenocarcinomas, BMC Cancer, 9 (2009) 336. [38] I.G. Tkalia, L.I. Vorobyova, V.S. Svintsitsky, S.V. Nespryadko, I.V. Goncharuk, N.Y. Lukyanova, V.F. Chekhun, Clinical significance of hormonal receptor status of malignant ovarian tumors, Exp Oncol, 36 (2014) 125-133.

[39] L. Paleari, S. Gandini, N. Provinciali, M. Puntoni, N. Colombo, A. DeCensi, Clinical benefit and risk of death with endocrine therapy in ovarian cancer: A comprehensive review and meta-analysis, Gynecol Oncol, 146 (2017) 504-513.

[40] T.J. Herzog, B. Pothuri, Ovarian cancer: a focus on management of recurrent disease, Nature Clinical Practice Oncology, 3 (2006) 604.

[41] S.L. Cooke, J.D. Brenton, Evolution of platinum resistance in high-grade serous ovarian cancer, Lancet Oncol, 12 (2011) 1169-1174.

[42] R. Beckerman, C. Prives, Transcriptional regulation by p53, Cold Spring Harb Perspect Biol, 2 (2010) a000935.

[43] M. Eren, A.E. Boe, E.A. Klyachko, D.E. Vaughan, Role of plasminogen activator inhibitor-1 in senescence and aging, Semin Thromb Hemost, 40 (2014) 645-651.

[44] D.R. Green, J.E. Chipuk, p53 and metabolism: Inside the TIGAR, Cell, 126 (2006) 30-32. 